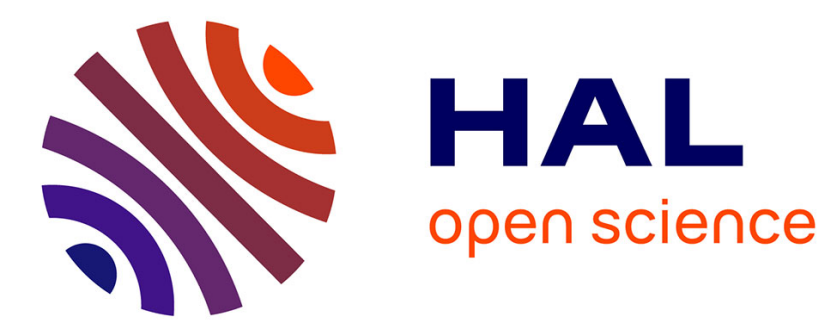

\title{
Contrôler une machine d'essai avec une caméra
}

Xavier Fayolle, Sylvain Calloch, François Hild

\section{To cite this version:}

Xavier Fayolle, Sylvain Calloch, François Hild. Contrôler une machine d'essai avec une caméra. Mechanics \& Industry, 2008, 9 (5), pp.447-457. 10.1051/meca/2009008 . hal-00392389

\section{HAL Id: hal-00392389 \\ https://hal.science/hal-00392389}

Submitted on 7 Jun 2009

HAL is a multi-disciplinary open access archive for the deposit and dissemination of scientific research documents, whether they are published or not. The documents may come from teaching and research institutions in France or abroad, or from public or private research centers.
L'archive ouverte pluridisciplinaire HAL, est destinée au dépôt et à la diffusion de documents scientifiques de niveau recherche, publiés ou non, émanant des établissements d'enseignement et de recherche français ou étrangers, des laboratoires publics ou privés. 


\title{
Contrôler une machine d'essai avec une
}

\section{caméra}

\author{
Xavier FAYOLLE Sylvain CALLOCH François HILD
}

LMT-Cachan, ENS de Cachan / CNRS UMR 8535 / Université Paris 6

61 avenue du Président Wilson, 94235 Cachan Cedex

Courriel : hild@1mt.ens-cachan.fr 


\title{
Contrôler une machine d'essai avec une caméra
}

\author{
Xavier FAYOLLE, Sylvain CALLOCH et François HILD
}

Résumé. Cet article discute d'une stratégie pour piloter un essai mécanique en utilisant la corrélation d'images. Un asservissement en cascade est développé en utilisant un algorithme de corrélation sub-pixel permettant l'évaluation d'une déformation moyenne dans une région d'étude pour piloter une machine électrohydraulique asservie. Une analyse a priori d'incertitude est validée a posteriori avec des mesures obtenues lors d'un essai mécanique. Le système développé est utilisé pour la conduite d'un essai de traction sur acier.

Mots-clés : Photomécanique, corrélation d'images, essai mécanique, asservissement

\begin{abstract}
It is proposed to discuss a strategy to control a mechanical test by using digital image correlation. A two-loop system is developed to integrate a sub-pixel correlation algorithm to evaluate the average strain of a region of interest and control a servo-hydraulic testing machine. An a priori uncertainty analysis is validated a posteriori with actual strain measurements. A tensile test is analyzed with the developed system.
\end{abstract}

Keywords: Photomechanics, image correlation, mechanical test, control 


\section{Introduction}

Les essais mécaniques consistent, par exemple, à analyser le comportement en traction / compression de divers matériaux. En particulier, les propriétés mécaniques telles que le module d'Young, le coefficient de Poisson, voire la limite d'écoulement ou la contrainte à rupture sont des quantités que l'on souhaite déterminer $[1,2]$. Ces quantités sont utilisées, par exemple, lors de simulations numériques pour le dimensionnement de structures mécaniques. Ces calculs font appel à des lois de comportement de plus en plus complexes qui nécessitent des investigations expérimentales toujours plus poussées. Par exemple, les essais mécaniques peuvent être analysés avec des moyens de mesure de champs (cinématiques ou de température) lorsqu'ils sont hétérogènes $[3,4]$. Un groupe de recherche (GDR) du CNRS traite des mesures de champs et de l'identification en mécanique des solides. Il a notamment pour tâche d'évaluer les performances des techniques de mesures optiques [5]. Il rassemble un certain nombre d'acteurs de laboratoires français qui utilisent et / ou développent des techniques de mesure de champs. Ces dernières sont utilisées dans des laboratoires universitaires et dans des environnements industriels $[6,7,8,9]$.

Pour le pilotage de ces essais mécaniques avec une histoire de déformation imposée, l'utilisation d'extensomètres ou de jauges de déformation est courante. Une alternative est l'utilisation d'images qui, par post-traitement (suivi de points [10], corrélation [11] ou stéréocorrélation [19]), peuvent être utilisées pour évaluer un champ de déplacement et par différentiation un champ de déformation. L'incertitude typique sur le déplacement $\rho_{u}$ d'un algorithme de corrélation est de l'ordre 
du centipixel pour des images codées sur 8 bits [13]. Suivant la longueur de jauge $L_{0}$ pour laquelle la déformation est évaluée [15], on peut atteindre des incertitudes en déformation de l'ordre de $\rho_{u} / L_{0}$ (soit $10^{-4}$ lorsque $L_{0}=100$ pixels, ou $10^{-5}$ lorsque $L_{0}=1000$ pixels). Cette dernière valeur est généralement suffisante lorsque l'on veut piloter une machine d'essai avec une histoire de déformation donnée. Ayant cet ordre de grandeur à disposition, le but de l'article est de montrer comment on peut procéder en pratique pour utiliser un algorithme de corrélation pour piloter une machine d'essai.

Le paragraphe 2 présente les éléments importants d'une machine électro-hydraulique asservie lorsque l'on veut la piloter. L'algorithme de corrélation utilisé est ensuite décrit au paragraphe 3. L'architecture et les paramètres de l'asservissement sont présentés au paragraphe 4. CORRELI ${ }^{\text {CONTROL }}$ prend en compte les spécificités, en termes de temps de cycle, de l'algorithme de corrélation. Les paramètres d'asservissement sont ensuite réglés. Un essai de traction sur acier est analysé au paragraphe 5. Le domaine élastique est tout d'abord commenté et une procédure de correction de déplacement hors-plan est introduite. Enfin, le domaine plastique est étudié à l'aide d'un logiciel de corrélation d'images.

\section{Piloter une machine électro-hydraulique}

Les principaux éléments qui constituent une machine d'essai électro-hydraulique sont rappelés en figure 1. Elle comprend un bâti, un (ou plusieurs) vérin(s), et une centrale hydraulique. De plus, des capteurs permettent la mesure de déplacement de vérin (par exemple, avec un LVDT) et d'effort. Enfin, des mesures 
externes par jauges ou extensomètres, voire thermocouples sont également possibles. Chacun de ces signaux peut être utilisé comme signal de retour pour piloter la machine de manière interne ou externe. Un asservissement analogique ou numérique permet un pilotage en boucle fermée beaucoup plus précis et robuste. Ainsi, un essai mécanique est piloté soit en déplacement de vérin imposé, soit en effort imposé, soit en déformation imposée en fonction du type d'investigation.

La figure 1 montre le cas d'un pilotage pour une histoire en déformation imposée. Le système d'asservissement comprend un capteur de déformation, un comparateur qui effectue la différence entre la consigne et le signal de la boucle de retour comprenant l'étage de PID et l'actionneur (i.e., la servovalve et le vérin hydraulique). Dans le cas présent, on souhaite remplacer le capteur de déformation par une caméra et un algorithme de corrélation d'images permettant le calcul de déplacements, puis de déformations. Ce choix conduit à deux difficultés qu'il s'agit de résoudre. La première est liée au fait que la caméra fournit des images qu'il faut ensuite post-traiter. La seconde est liée au fait qu'il faut un certain temps pour passer d'images à l'information pertinente, ici une déformation moyenne. Comme il sera montré plus bas, le temps de post-traitement de la version actuelle n'est pas compatible avec la bande passante d'une machine conventionnelle. On ne peut pas simplement remplacer la jauge ou l'extensomètre par une caméra et un algorithme de corrélation. Une architecture particulière d'asservissement est nécessaire. 


\section{Algorithme de corrélation et performances}

Un algorithme de corrélation d'images est implémenté pour piloter une machine d'essais à partir d'images. Comme montré plus haut, une histoire de déformation est l'information d'entrée (i.e., la consigne) qu'il s'agit de comparer avec une mesure réelle sur éprouvette. Afin de pouvoir concevoir le système d'asservissement, deux paramètres importants doivent être connus. Le premier concerne l'incertitude de mesure et le second le temps de cycle de l'algorithme de corrélation.

Pour un algorithme de corrélation, l'incertitude de mesure en déformation dépend essentiellement de trois paramètres. Le premier concerne la qualité de l'image (i.e., la texture), le second la taille $\ell$ des zones d'étude (qui conditionne la résolution spatiale en déplacement), et enfin la séparation $\delta$ entre zones d'études pour le calcul de la déformation [16]. En effet, l'utilisateur définit sur le couple d'images à analyser (images correspondant à l'état initial et déformé) la région d'étude (zone virtuelle qui sert de base de mesure dans l'image de référence). Dans celle-ci, il considère plusieurs zones d'études qu'il s'agit d'apparier entre une première image et une seconde. Cet appariement étant fait, il est alors possible de déterminer le déplacement de chaque zone d'étude. En post-traitement on peut alors calculer, par exemple la déformation moyenne observée sur un ensemble de zones d'étude. L'appariement entre images initiale et déformée est obtenu en utilisant un critère de ressemblance entre zones d'études (produit de corrélation [17]). Une première évaluation en nombre entier de pixels est obtenue en cherchant le maximum de la fonction de corrélation. Une interpolation biparabo- 
lique au voisinage de ce maximum permet d'obtenir une estimation sub-pixelle du déplacement [18]. D'autres approches sont également possibles par interpolation des niveaux de gris [12]. Des itérations successives permettent d'affiner cette évaluation en utilisant la propriété de décalage / modulation des transformées de Fourier [13]. Ce dernier point est particulièrement important lors du pilotage, puisque des niveaux très faibles de déformation doivent être mesurés. En pratique, trois itérations sub-pixelles sont nécessaires pour obtenir une variation inférieure à $5 \times 10^{-3}$ pixel avec un algorithme basé sur des transformées de Fourier rapides. Dans le cas présent, les déplacements mesurés entre deux images sont très faibles. Ainsi, l'hypothèse de déplacement constant par zone d'étude est tout à fait raisonnable.

Les incertitudes en déformation sont évaluées de la manière suivante. On considère une image de référence de la région d'étude de l'éprouvette dont on souhaite contrôler les déformations longitudinales. Cette image est décalée artificiellement par transformée de Fourier à l'aide de la propriété de décalage / modulation par incréments de 0.1 pixel dans chaque direction. Pour chaque valeur de déplacement, l'écart quadratique moyen est évalué et correspond à l'incertitude type. Celle-ci est évaluée pour chaque valeur imposée entre 0 et 1 pixel. La valeur moyenne est ensuite calculée et est appelée incertitude en déplacement $\rho_{u}$. Cette dernière est estimée pour différente tailles $\ell$ de zones d'étude. Pour chaque décalage imposé, le champ de déformation est obtenu par différences finies centrées. Les incertitudes sont alors calculées de la même manière que pour les déplacements. La valeur moyenne, $\rho_{\epsilon}$ est appelée incertitude en déformation.

Afin d'avoir des mesures de déplacement indépendantes, le décalage $\delta$ entre 
zones d'études consécutives est pris égal à la taille des zones $\ell$. La première étape est appliquée à l'image considérée. La figure 2 montre l'évolution de l'incertitude en déplacement $\rho_{u}$ en fonction de $\ell$. Pour des tailles supérieures à 16 pixels, une loi de puissance décrit très raisonnablement cette évolution

$$
\rho_{u}=\frac{A^{\alpha+1}}{\ell^{\alpha}}
$$

avec $A=1.43$ pixels (et $\alpha=1.5$ ). On montre ainsi que plus la résolution spatiale $(\ell)$ est importante, plus l'incertitude est faible. On aboutit au compromis entre incertitude et résolution spatiale. Cependant, augmenter la résolution spatiale revient également à augmenter le temps de calcul pour estimer un déplacement. Le choix s'est porté sur une taille $\ell$ égale à 64 pixels qui conduit à une incertitude en déplacement de $5 \times 10^{-3}$ pixel.

L'incertitude en termes de déformation est évaluée. Dans le cas présent un algorithme de différences finies centrées est utilisé de telle manière que la résolution spatiale en déformation est égale à $2 \delta$. Par analyse dimensionnelle on obtient

$$
\rho_{\epsilon}=B \frac{\rho_{u}}{2 \delta}
$$

avec $B=1.3$. Ce résultat montre que l'incertitude en déformation dépend des deux paramètres de corrélation, explicitement de $\delta$ et implicitement de $\ell$ par l'intermédiaire de $\rho_{u}$ [cf. équation (1)]. L'équation (2) est retrouvée dans le cas présent, en particulier un exposant de -2.5 pour la dépendance en taille $\ell$ de par le fait que $\delta=\ell$ pour le cas étudié.

Comme indiqué précédemment, l'incertitude en déformation dépend de deux paramètres. Le premier, la taille $\ell$, a été fixé suite à un compromis entre taille de 
zone d'étude et incertitude en déplacement. Il reste maintenant à fixer le décalage $\delta$. Plus celui-ci est grand, plus l'incertitude en déformation est faible [cf. équation (2)]. Dans l'analyse qui suit, on va considérer $3 \times 3$ zones d'étude (Fig. 7) pour lesquelles la déformation moyenne est calculée par interpolation bilinéaire. Dans ce cas, la constante $B$ décroît $(B=\sqrt{2 / 3} \approx 0.8$ [14]). Une incertitude maximale de $10^{-5}$ est obtenue pour une longueur de jauge $L_{0}$ (i.e., un décalage entre zones d'étude de $L_{0} / 2$ ) au moins égale à 400 pixels.

\section{Asservissement implémenté}

Pour piloter un essai avec une histoire de déformation donnée, deux options sont possibles. La première (et la plus facile) est d'utiliser le conditionneur interne de la machine d'essai. Ceci est possible lorsqu' on utilise un extensomètre ou une jauge de déformation. La fréquence d'échantillonnage doit alors être plus importante que la bande passante de la machine d'essai. Lorsque cette condition n'est pas satisfaite, une architecture en cascade est implémentée. La machine est alors pilotée à l'aide d'une boucle rapide (avec un contrôle en déplacement), elle-même pilotée par une boucle plus lente utilisant, par exemple un algorithme de corrélation. Pour des systèmes asservis échantillonnés, lorsque la vitesse d'échantillonnage $f_{s}$ est supérieure à 30 fois celle de sa bande passante $f_{b w}$, on peut appliquer les règles des systèmes analogiques avec une très bonne approximation. Dans le cas général, lorsque l'inéquation

$$
5 f_{b w} \leq f_{s} \leq 25 f_{b w}
$$


est satisfaite [20], on doit travailler avec des transformées en $z$ pour modéliser le système. Pour des vitesses d'échantillonnage inférieures à $3 f_{b w}$, une procédure d'asservissement est périlleuse de par le risque de négliger des variations rapides du système. On montrera plus bas que le temps nécessaire à l'acquisition des images et au traitement de celles-ci par corrélation pour déterminer la déformation moyenne recherchée est de l'ordre de $6 \mathrm{~s}$. Ainsi, la fréquence d'échantillonnage est de l'ordre de $0.15 \mathrm{~Hz}$. Pour que le système puisse être pilotable, il faudrait une bande passante de $0.03 \mathrm{~Hz}$, ce qui est mille fois plus faible que les valeurs couramment rencontrées (i.e., $30 \mathrm{~Hz}$ environ). Ceci a pour conséquence d'imposer une architecture en cascade (Fig. 3).

Ce système doit maintenant être caractérisé de manière à éviter toute réponse erratique qui pourrait entraîner la rupture d'un échantillon. Ceci est obtenu en limitant l'incrément de déplacement imposé. On aboutit alors à un système nonlinéaire. De plus, une réponse stable avec une erreur statique nulle est également souhaitée (recours à un correcteur intégral). La figure 3-b montre le diagramme bloc du système. La boucle d'asservissement en déformation inclut une boucle interne rapide qui est pilotée en déplacement. Un ordinateur permet l'acquisition d'images et le système d'asservissement externe. L'ensemble a été développé dans l'environnement LabVIEW ${ }^{\mathrm{TM}}$ [21]. La boucle externe est pilotée de manière statique, i.e., la consigne est imposée par pas, et entre deux incréments, la réponse de la machine converge vers la consigne de manière que l'erreur statique devienne inférieure à l'incertitude en déformation. La consigne en déformation est ainsi définie comme une suite de petits incréments.

Les différents paramètres du système doivent être réglés. Pour choisir le gain 
$K_{i}$ de l'intégrateur (Fig. 3-b), on a besoin d'estimer le gain statique en boucle ouverte qui dépend de l'incertitude en déformation de l'algorithme de corrélation. Dans le cas présent, l'erreur maximale est imposée à $2 \times 10^{-5}$, i.e., deux fois l'incertitude en déformation (cf. paragraphe 3). L'incrément de déplacement $\delta U$ est tel que, $\delta U=2 \rho_{\epsilon} L_{g}$, où $L_{g}$ est la longueur de la zone utile de l'éprouvette. La carte d'acquisition de l'ordinateur ayant un convertisseur numérique / analogique (CNA) codant des tensions de $\pm 10 \mathrm{~V}$ sur 16 bits, la résolution de ce dernier est de $0.3 \mathrm{mV}$ (i.e., $20 / 2^{16}$ ). Un incrément élémentaire de CNA se traduira par un incrément de déplacement $\delta U$, ce qui conduit pour une amplitude de $10 \mathrm{~V}$ à une amplitude de déplacement $\Delta U=2 \times 10^{-5} \times 10 / 3 \times 10^{-4} L_{g}$ ou encore

$$
\Delta U \leq 0.65 L_{g}
$$

Dans le cas présent, $L_{g}=90 \mathrm{~mm}$ (Fig. 7), soit une amplitude de déplacement de $59 \mathrm{~mm}$ pour une amplitude de tension de $10 \mathrm{~V}$. Une valeur maximale estimée de $12.5 \mathrm{~mm}$ vérifie l'inéquation précédente. Le gain $G_{1}$ de la machine d'essai est alors de $1.25 \mathrm{~mm} / \mathrm{V}$. Le réglage du gain de l'intégrateur devrait être déterminé par simulation avec un logiciel adapté après avoir modélisé et calculé les fonctions de transfert (en $z$ ) du système. Dans le cas présent, le fonctionnement quasi statique conduisant à un système à bande passante faible, on peut s'affranchir des transformées en $z$ et utiliser les transformées de Laplace pour le caractériser. Si le PID du conditionneur de la machine d'essai est réglé de telle manière qu'il n'y a pas de dépassement, alors la fonction de transfert de la machine pilotée en déplacement peut être décrite par un système du premier ordre $G_{1} /\left(1+\tau_{m} s\right)$, avec $\tau_{m} \approx 20 \mathrm{~ms}$. Ceci est possible car le système a un pôle dominant (i.e., une atténuation en gain 
de $-20 \mathrm{~dB}$ par décade après la fréquence de coupure). Cependant, l'ordre du système étant supérieur à 1 , on observe un déphasage supérieur à $90^{\circ}$. Ce dernier est pris en compte pour le calcul de la marge de phase. Il y a une fonction de transfert additionnelle de gain $G_{0}=1 / L_{g}$ pour permettre un pilotage en déformation de l'éprouvette. La caméra prend des images à une cadence maximale de 12 images par seconde. Un retard $\tau_{0}$ de 83 ms est ainsi observé. Le système optique se comporte donc comme un filtre passe-bas (idéal) avec une fréquence de coupure de $12 \mathrm{~Hz}$ et un décalage $\tau_{0}$. Cette fonction de transfert n'est pas considérée car la fréquence de coupure est supérieure de deux ordres de grandeur par rapport à celle du système de mesure (i.e., $0.15 \mathrm{~Hz}$ ). Comme indiqué plus haut, la fréquence d'échantillonnage est au plus égale à $0.03 \mathrm{~Hz}\left(\right.$ i.e., $\left.\tau_{s} \approx 6 \mathrm{~s}\right)$. Un bloqueur d'ordre 0 est ajouté dans la boucle afin de rendre compte de ce phénomène. Sa fonction de transfert approchée s'écrit: $H_{0}(s)=H_{0} \exp \left(-\tau_{s} s / 2\right)$, avec $H_{0}$ un nombre réel tel que $\left|H_{0}\right| \leq 1$ (pour $\omega=1 \mathrm{rad} / \mathrm{s}$ ). Par la suite, on considère que $\left|H_{0}\right|=1$. Ceci permet d'obtenir une borne inférieure de la marge de phase. L'intégrateur a une fonction de transfert: $K_{i} / s$. La fonction de transfert en boucle ouverte du système complet s'écrit $H_{0} K_{i} G_{0} G_{1} \exp \left(-\tau_{s} s / 2\right) / s\left(1+\tau_{m} s\right)$. La figure 4-a montre le gain du système en fonction de la pulsation et celui de la boucle interne. Lorsque le gain en boucle ouverte est pris égal à 1 (i.e., $K_{i} G_{0} G_{1}=1$ ), le système asservi est stable, même sur-amorti, avec une marge de phase de $75^{\circ}$ (Fig. 4-b). Le décalage de phase induit par la fréquence d'échantillonnage est également considéré lors du calcul de la phase du système. Il s'ensuit que le gain $K_{i}$ est égal à $126 \mathrm{~V}$. Cette approche, pragmatique, permet d'obtenir un système qui remplit le cahier des charges imposé. Son optimisation, non traitée ici, nécessitera des simulations 
plus poussées.

La figure 5 détaille l'algorithme implémenté. Pour des raisons de sécurité, il est souhaité que des incréments de déformation supérieurs à $2 \times 10^{-4}$ soient écrêtés. L'erreur maximale du système asservi est imposée à $14 \mathrm{mV}$ avec les paramètres choisis. Les incréments du convertisseur sont ainsi limités dans une plage de $\pm 14 \mathrm{mV}$, lorsque $K_{i}=126 \mathrm{~V}$. L'équation aux différences finies de l'intégrateur s'écrit

$$
u(k)=u(k-1)+\operatorname{clip}\left(T K_{i} \varpi(k)\right)
$$

où $u($.$) est la sortie de l'asservissement, k$ le pas considéré, clip(.) la limite à $14 \mathrm{mV}, T$ la période d'échantillonnage, $K_{i}$ le gain de l'intégrateur et $\varpi($.$) le$ signal d'erreur. Le programme, CORRELI ${ }^{\mathrm{CONTROL}}$, a été testé avec les valeurs indiquées ci-dessus. La réponse à un échelon est déterminée (Fig. 6). Environ 16 points de mesure sont nécessaires lors d'un intervalle de temps de $100 \mathrm{~s}$, soit une période d'échantillonnage de $6 \mathrm{~s}$ environ. Les fluctuations de déformations sont inférieures à celles liées à l'incertitude de mesure. Ceci est lié à l'intégration effectuée après le calcul de la déformation (Fig. 3-b) et qui permet de réduire les fluctuations. Ceci montre la robustesse du système asservi vis-à-vis des fluctuations induites par l'algorithme de corrélation. Lorsque la réponse à un échelon est approchée par celle d'un système du premier ordre, une constante de temps $\tau$ de 75 s est identifiée. Ceci montre qu'aucun dépassement n'est observé, ce qui permet d'éviter des ruptures prématurées d'éprouvettes. D'autre part, une marge de phase de l'ordre de $90^{\circ}$ est obtenue, en accord avec l'analyse a priori (Fig. 4-b). Le programme CORRELI ${ }^{\mathrm{CONTROL}}$ est maintenant complètement caractérisé. Il 
s'agit maintenant de l'utiliser sur un cas réel.

\section{Pilotage d'un essai de traction}

Un essai de traction est conduit avec CORRELI ${ }^{\text {CONTROL }}$ sur un acier de construction. Afin de pouvoir rendre compte d'éventuels déplacements hors-plan, 3 capteurs de déplacement (LVDT) ont été utilisés. Deux rosettes sont collées de part et d'autre de l'éprouvette pour permettre une estimation a posteriori du niveau d'incertitude du système de pilotage. La figure 7 montre la répartition des différents capteurs ainsi que la région d'étude choisie avec les $3 \times 3$ zones d'études pour la détermination de la déformation moyenne. La caméra utilisée ici a une résolution de $1024 \times 1280$ pixels et utilise un codage sur 12 bits [22]. Cependant, la dynamique réelle est de 10.5 bits de par le bruit de lecture et d'obscurité.

\subsection{Première analyse de l'essai}

La figure 8 montre l'histoire de déformation imposée à l'éprouvette. Elle consiste en plusieurs phases de charge et de décharge tout d'abord dans le domaine élastique puis dans le domaine plastique. Les décharges s'arrêtent pour un effort de $7.5 \mathrm{kN}$ environ. De cette figure, on peut conclure que le système de pilotage est opérationnel et que les réglages effectués précédemment sont validés. L'analyse des résultats peut ainsi être effectuée.

La figure 9 montre le résultat des mesures de déformation par jauges et corrélation d'images. Les tendances générales sont cohérentes. Cependant, on note, d'une part, une différence de pentes dans le domaine élastique entre les signaux 
obtenus par les deux moyens de mesure de déformation. D'autre part, dans le domaine plastique, le signal délivré par la jauge est inexploitable contrairement aux informations obtenues par corrélation d'images. Ces deux aspects sont analysés par la suite.

Dans le domaine élastique, on observe une raideur longitudinale "apparente" obtenue à partir des déformations mesurées par corrélation d'images supérieure à celle déterminée à partir des signaux de jauge. En ce qui concerne la raideur transverse, des tendances opposées sont observées. L'analyse des résultats obtenus à partir des déformations relevées par les jauges montre que le module d'Young du matériau est de l'ordre de $203 \mathrm{GPa}$, le coefficient de Poisson de 0.27 et la limite d'écoulement aux environs de $280 \mathrm{MPa}$. En ce qui concerne les estimations à partir des données obtenues par corrélation d'images, un module d'Young apparent de 336 GPa est identifié (Fig. 10). Bien qu'un léger effet de flexion parasite soit présent, ceci ne permet pas d'expliquer une telle différence. Ceci est confirmé par l'obtention d'un coefficient de Poisson apparent de 1.09 (valeur physiquement non réaliste). A partir des images acquises au cours de l'essai, un post-traitement avec un logiciel de corrélation d'images validé de nombreuses fois $[23,13,16]$ conduit exactement aux mêmes résultats. L'algorithme de corrélation mis en œuvre n'est pas en cause.

L'analyse des données mesurées à l'aide des trois capteurs de déplacement permet d'émettre l'hypothèse d'un effet parasite causé par des déplacements horsplan. En effet, l'éprouvette a été usinée à partir d'une tôle en acier et sa forme initiale n'était pas parfaitement plane. D'autre part, la rigidité du montage n'était pas optimale vis-à-vis de la taille de l'éprouvette testée. Ceci peut expliquer l'ampli- 
tude des déplacements hors-plan mesurés (Fig. 11). Ceux-ci varient linéairement avec la contrainte moyenne. Il s'agit donc d'analyser l'effet d'un déplacement hors-plan sur la mesure du déplacement dans le plan et de proposer une procédure de correction.

\subsection{Déformations virtuelles induites par un déplacement hors- plan}

L'objet de ce paragraphe est de montrer qu'un déplacement hors-plan induit des déformations "virtuelles" dans le plan. Considérons un objet $A B$ qui est imagé à l'aide d'un objectif décrit par une lentille $L$ sur un capteur CCD pour obtenir l'image $A^{\prime} B^{\prime}$ (Fig. 12). Si l'objet $A B$ se déplace d'une quantité $w>0$ dans la direction de l'axe optique pour prendre la position $A^{*} B^{*}$, alors l'image $A^{\prime} B^{\prime *}$ devient plus petite et floue. Cette variation de grandissement se traduit par une déformation uniforme et isotrope lorsque, ni les aberrations optiques, ni la défocalisation ne sont pas prises en compte. Cet effet conduit ainsi à une diminution de la déformation "apparente" par rapport à la déformation vraie pour la direction longitudinale, d'où une sur-estimation du module d'Young. A contrario, dans la direction transversale, cet effet conduit à une sur-estimation de la déformation et également du coefficient de Poisson apparent (Fig. 13). Une procédure de correction est implémentée pour prendre en compte l'effet des déplacements hors-plan. 


\subsection{Stratégie de correction}

La déformation apparente, i.e., mesurée par corrélation d'images, est la combinaison de deux déformations; la déformation vraie et celle (virtuelle) induite par un déplacement hors-plan. Sachant que le déplacement hors-plan est lui-même proportionnel à la contrainte moyenne $\sigma$ (Fig. 11), on en déduit que la déformation longitudinale apparente $\epsilon_{a}$ s'écrit

$$
\epsilon_{a}=\frac{\sigma}{E_{a}}=\frac{\sigma}{E}+\frac{\sigma}{K_{v}}
$$

où $E_{a}$ est le module d'Young apparent, $E$ est le module d'Young du matériau, et $K_{v}$ le facteur de proportionnalité entre la déformation induite par le déplacement hors-plan et la contrainte appliquée. De cette relation et des résultats observés, on en déduit que

$$
\frac{1}{K_{v}}=\frac{1}{E_{a}}-\frac{1}{E}
$$

soit $K_{v}=513 \mathrm{GPa}$. Ceci permet de recaler les courbes de déformations longitudinales. A titre de validation, on peut appliquer le même type de correction aux déformations transverses pour relier les raideurs transverses apparente $E_{a t}$ et réelle $E_{t}$

$$
\frac{1}{E_{t}}=\frac{1}{E_{t a}}-\frac{1}{K_{v}}
$$

On obtient $E_{t}=770 \mathrm{GPa}$. Cette valeur est très proche de celle estimée par la jauge (i.e., $752 \mathrm{GPa}$ ). Une erreur de $2.4 \%$ est ainsi obtenue. Ce résultat permet de valider la procédure de correction utilisant l'information obtenue par les jauges. Le coefficient de Poisson $(\nu \approx 0.27)$ déduit de $E_{t}=-E / \nu$ est très proche de la valeur obtenue à partir des mesures par jauges (0.27). La procédure de correction est ainsi validée. 


\subsection{Correction utilisant l'optique géométrique}

La correction précédente n'est possible que dans la mesure où le module d'Young du matériau est connu. En pratique, ceci n'est pas nécessairement le cas. Il est alors nécessaire de développer une méthode plus générale de correction. On utilise des résultats d'optique géométrique [24]. Pour cela, on suppose que l'objectif peut être modélisé par une seule lentille convergente. L'effet d'un déplacement hors plan est décrit sur la figure 12. Le grandissement transverse $m$ d'une lentille s'exprime comme le rapport de la taille de l'objet sur le plan image sur la taille physique

$$
m=\frac{A^{\prime} B^{\prime}}{A B}
$$

Par les relations classiques d'optique géométrique, on a: $A B / O A=A^{\prime} B^{\prime} / O A^{\prime}$, soit $m=O A^{\prime} / O A$. La taille de l'objet $A B$ sur le plan image est $m A B$. Un déplacement hors-plan $w$ induit une variation de grandissement $m^{*}$

$$
m^{*}=\frac{A^{\prime} B^{\prime *}}{A B}=m\left(1+\frac{w}{D_{0}}\right)^{-1}
$$

où $D_{0}$ est la distance (frontale) de l'objet à la lentille. La déformation (virtuelle) $\epsilon_{v}$ induite par un déplacement hors-plan $w$ s'écrit

$$
\epsilon_{v}=\frac{B^{\prime} B^{\prime *}}{A^{\prime} B^{\prime}}=-\frac{w}{D_{0}}\left(1+\frac{w}{D_{0}}\right)^{-1} \approx-\frac{w}{D_{0}}
$$

L'équation (11) montre qu'en première approximation la déformation virtuelle dépend linéairement du déplacement $w$. Si ce dernier varie linéairement avec la contrainte moyenne (cf. Fig. 11), alors $\epsilon_{v}$ varie linéairement avec $\sigma$ et l'on retrouve ainsi le facteur de proportionnalité $K_{v}$ introduit ci-dessus. En considérant le déplacement moyen déterminé à partir des trois capteurs de déplacement, on 
obtient une variation de déplacement hors plan de $0.14 \mathrm{~mm}$ pour une variation de contrainte de $160 \mathrm{MPa}$. En supposant que $D_{0}$ est égale à la distance frontale (445 mm), on identifie la constante $K_{v}$ à partir de

$$
\epsilon_{v}=\frac{\sigma}{K_{v}}
$$

qui vaut $509 \mathrm{GPa}$. Cette dernière est en très bon accord avec celle identifiée à partir des mesures de jauge (i.e., $513 \mathrm{GPa}$ ). La procédure de correction est considérée comme complètement validée. On obtient ainsi la courbe contrainte / déformations après correction (Fig. 14).

\subsection{Incertitude en déformation}

Dans le domaine élastique, et après correction, on peut estimer les incertitudes en déformation à la fois pour la jauge et la corrélation d'images. Celle-ci est obtenue à partir de l'écart quadratique moyen par rapport à une réponse purement linéaire. Dans le cas présent (Fig. 15), on obtient un rapport 2.3 entre les incertitudes obtenues à l'aide de mesures par corrélation d'images et celles de la jauge. Ceci montre que l'on peut atteindre des niveaux de performance comparables aux jauges avec le système présenté ici. On notera que si l'on augmente la dynamique du capteur, sans trop augmenter le niveau de bruit, on peut espérer diminuer les incertitudes [13]. Il en est de même si l'on augmente la résolution du capteur; ceci permet d'augmenter la taille de la région d'étude et ainsi de diminuer les incertitudes en déformation [cf. équations (1) et (2)]. On notera enfin que l'estimation a priori de niveau d'incertitude $\left(\approx 10^{-5}\right)$ est validée a posteriori, puisqu'on obtient une valeur de $1.1 \times 10^{-5}$ pour $\rho_{\epsilon}$. Ceci permet également de valider le niveau 
d'incertitude en déplacement $\rho_{u} \approx 5 \times 10^{-3}$ pixel dans le cas présent.

\subsection{Etude de la réponse plastique}

La dernière partie de l'étude concerne le domaine plastique qui n'a pas pu être analysé avec les données des jauges. Il est probable qu'elles aient été endommagées. Pour corroborer cette hypothèse, les images stockées lors de l'essai sont analysées par un logiciel de corrélation d'images standard [23, 13]. Afin d'obtenir un plus grand nombre d'informations, les paramètres de corrélation sont choisis de telle manière que $\ell=32$ pixels et $\delta=32$ pixels. L'incertitude sur les déplacements mesurés est un peu plus grande (Fig. 2), mais reste à des niveaux acceptables. L'analyse des champs de déplacements mesurés dans le domaine élastique (Fig. 16) correspond à un essai de traction homogène. Par contre, dans le domaine plastique, on observe très nettement des hétérogénéités fortes (i.e., localisation) près des deux coins à droite de la région d'étude. Ceci est la manifestation cinématique d'une réponse élastique-parfaitement plastique observée macroscopiquement. Localement, les champs de déplacement et de déformation sont très hétérogènes. De par l'endroit où a été collée la jauge (Fig. 7), on peut conclure que la zone de localisation inférieure traverse la jauge et a pu l'endommager ou la décoller partiellement. On montre ainsi que, dans le cas présent, il n'aurait pas été possible de piloter de manière satisfaisante l'essai avec les jauges de déformation. 


\section{Conclusion}

Il a été montré que la corrélation d'images peut être utilisée pour piloter un essai mécanique avec une machine électro-hydraulique asservie. Un logiciel, CORRELI ${ }^{\mathrm{CONTROL}}$, a été développé de manière à prendre en compte plusieurs aspects spécifiques. De par le temps (relativement long) de stockage et de traitement des images, un cycle de pilotage dure environ 6 s. Ceci nécessite une architecture en cascade pour pouvoir piloter de manière robuste un asservissement de machine d'essais mécaniques. Seuls des essais quasi statiques sont actuellement envisageables avec ce type d'implémentation. Les paramètres d'asservissement sont peu nombreux et facilement réglables. Ils pourront être optimisés dans un futur proche, en particulier en termes de niveau d'écrêtement.

Les résultats obtenus à partir de l'essai mécanique présenté montrent la faisabilité du système. De par les conditions expérimentales, il a été nécessaire de développer une procédure de correction de déplacement hors-plan. Elle a pu être complètement validée dans le cas présent. Avec la caméra utilisée (résolution = $1024 \times 1280$ pixels, dynamique $=10.5$ bits $)$ une incertitude en déplacement de l'ordre de $5 \times 10^{-3}$ pixel et une incertitude en déformation de $1.1 \times 10^{-5}$ ont été obtenues. Cette dernière valeur est proche de celle observée sur le signal de jauges. Ce niveau de performance est suffisant pour tester un grand nombre de matériaux de l'industrie mécanique. Enfin, le post-traitement des images par un code de corrélation standard a permis de comprendre la raison pour laquelle il n'aurait pas été possible de piloter dans de bonnes conditions l'essai analysé. Des lignes de localisation sont observées à différents endroits de l'éprouvette. 
Le développement de ces techniques d'analyse d'images ne vient pas simplement améliorer la productivité ou l'efficacité du mécanicien devant sa machine d'essai. Il remet en cause la conception même des essais. En effet, l'homogénéité de l'essai ou sa simplicité n'est plus un impératif, et en conséquence des essais hétérogènes peuvent être imaginés, non pour le plaisir d'une complication additionnelle, mais par souci d'efficacité, de manière à pouvoir appréhender sur un seul essai le plus d'informations utiles sur le comportement mécanique du milieu. Ceci n'est pas une simple vue de l'esprit car une sollicitation hétérogène donne beaucoup plus d'informations, et permet une formulation du problème d'identification des paramètres constitutifs mieux conditionnée. Cela est à même de bouleverser complètement la stratégie de conception des essais mécaniques.

L'intégration de techniques d'analyse inverse ou de recalage par modélisation numérique $[5,25]$ venant directement interagir avec les mesures de champs vient compléter ce dispositif en permettant des interactions fructueuses entre expérience et calcul, pourquoi pas, dans un futur proche, en temps réel. On peut alors rêver de la machine d'essai intelligente qui vienne appliquer la sollicitation utile pour acquérir l'information manquante dans le processus d'identification des propriétés recherchées.

\section{Remerciements}

Les auteurs remercient Yvan Bonnassieux pour les remarques très utiles faites lors de la rédaction de cet article. 


\section{Références}

[1] M.R. Moreau, ed., Essais et moyens d'essais au laboratoire et dans l'industrie, Tec \& Doc - Lavoisier, Paris (France), 1982.

[2] ISO, Matériaux métalliques - Essai de traction à température ambiante, International Organization for Standardization, Genève (Suisse), 1998.

[3] A. Lagarde, ed., Advanced Optical Methods and Applications in Solid Mechanics, Kluwer, Dordrecht (Pays-Bas), 2000.

[4] Y. Berthaud, F. Hild, P. Jacquot, P. Smigielski, Y. Surrel, Identification $d u$ comportement des matériaux et de la rupture des structures à l'aide de méthodes optiques, Cours IPSI, Paris (France), 2003.

[5] GDR-2519, Mesures de champs et identification en mécanique des solides, www.ifma.fr/lami/gdr2519/, 2003-2010.

[6] Y. Berthaud, D. Paraskevas, M. Taroni, eds., Photomécanique 95, GAMAC, Paris (France), 1995.

[7] Y. Berthaud, M. Cottron, F. Morestin, P. Moucheront, M. Taroni, eds., Photomécanique 98, GAMAC, Paris (France), 1998.

[8] Y. Berthaud, M. Cottron, J.-C. Dupré, F. Morestin, J.-J. Orteu, V. Valle, eds., Photomécanique 2001, GAMAC, Paris (France), 2001.

[9] Y. Berthaud, P. Gaborit, M. Grédiac, J.-J. Orteu, L. Robert, T. Sentenac, eds., Photomécanique 2004, EMAC, Albi (France), 2004.

[10] C. G'Sell, J.-M. Hiver, A. Dahnoun, A. Souahi, Video-Controlled Tensile Testing of Polymers and Metals Beyond the Necking Point, J. Mat. Sci. 27 (1992) 5031-5039. 
[11] T.C. Chu, W.F. Ranson, M.A. Sutton, W.H. Peters, Applications of DigitalImage-Correlation Techniques to Experimental Mechanics, Exp. Mech. 3 (1985) 232-244.

[12] H.W. Schreier, J.R. Braasch, M.A. Sutton, Systematic errors in digital image correlation caused by intensity interpolation, Opt. Eng. 39 (2000) 29152921.

[13] J.N. Périé, S. Calloch, C. Cluzel, F. Hild, Analysis of a Multiaxial Test on a C/C Composite by Using Digital Image Correlation and a Damage Model, Exp. Mech. 42 (2002) 318-328.

[14] ISO (1995) Guide to the Expression of Uncertainty in Measurements (GUM). International Organization for Standardization, Geneva (Switzerland).

[15] L. Allais, M. Bornert, T. Bretheau, D. Caldemaison, Experimental characterization of the local strain field in a heterogeneous elastoplastic material, Acta Met. Mater. 42 (1994) 3865-3880.

[16] S. Bergonnier, F. Hild, S. Roux, Strain heterogeneities in tension and compression tests on mineral wool samples, J. Strain Analysis 40 (2005) 185197.

[17] M.A. Sutton, W.J. Wolters, W.H. Peters, W.F. Ranson, S.R. McNeill, Determination of Displacements Using an Improved Digital Correlation Method, Im. Vis. Comp. 1 (1983) 133-139.

[18] Y. Berthaud, J. Scholz, J. Thesing, Méthodes optiques et acoustiques de mesures des caractéristiques mécaniques, Proceedings Colloque national ME- 
CAMAT "Mécanismes et mécanique des grandes déformations", (1996) 7780.

[19] D. Garcia, J.-J. Orteu, Mesure de formes et de déformations 3D par stéréocorrélation: application sur des tôles minces embouties, Actes Nouveaux moyens optiques pour l'industrie, SFO, Club Contrôles et mesures optiques pour l'industrie, (1999) 177-184.

[20] M. Rivoire, J.-L. Ferrier, Cours d'automatique. Commande par calculateuridentification, Eyrolles, Paris (France), 1990.

[21] National Instrument, LABVIEW $W^{T M}$ version 5.1, user's manual (www.ni.com), 1999.

[22] PCO, PixelFly High Performance Digital CCD Camera System, www.pco.de, 2000.

[23] F. Hild, B. Raka, M. Baudequin, S. Roux, F. Cantelaube, Multi-Scale Displacement Field Measurements of Compressed Mineral Wool Samples by Digital Image Correlation, Appl. Optics IP 41 (2002) 6815-6828.

[24] J.C. Hild, Elément de cours et expériences d'optique, Centre de Publication Universitaire, Tunis (Tunisie), 2000.

[25] M. Bonnet, A. Constantinescu, Inverse problems in elasticity, Inverse Problems 21 (2005) R1-R50. 


\section{Table des figures}

1 Schéma d'une machine electro-hydraulique asservie avec un pilotage en déformation. . . . . . . . . . . . . . . . . . . . . 29

2 Incertitudes en déplacement et en déformation en fonction de la taille des zones d'étude. Les symboles pleins sont des résultats de corrélation. Les droites en trait continu correspondent à une interpolation en loi de puissance $(-1.5)$ et en traits pointillés à une loi avec un exposant $-2.5 \ldots \ldots$. . . . . . . . . . . 30

3 a- Schéma d'une implantation d'un asservissement en cascade utilisant la corrélation d'images pour le pilotage d'une machine électro-hydraulique asservie. b- Diagramme bloc d'un asservissement en cascade. Les points A et B correspondent aux signaux de la figure $6 \ldots \ldots \ldots \ldots \ldots$

4 Diagrammes de Bode pour le système complet et la boucle intérieure (machine d'essai pilotée en déplacement). a- Gain en fonction de la pulsation. b- Phase en fonction de la pulsation. Le déphasage induit par l'échantillonnage est également montré. . . . . 32

5 Organigramme de l'asservissement. ............ 33

6 Réponse à un échelon du système et description par un premier ordre avec une constante de temps $\tau=75$ s. . . . . . . . . . . 34

7 Géométrie de l'éprouvette et localisation des différents systèmes de mesure. . . . . . . . . . . . . . . . 35 
8 Déformation longitudinale en fonction du temps pour l'essai analysé. ............................ 36

9 Courbe contrainte / déformations obtenue à partir de mesures avec des jauges de déformation et par corrélation d'images. . . . . . 37

10 Courbe contrainte / déformation longitudinale dans le domaine élastique obtenue à partir de mesures avec des jauges de déformation et par corrélation d'images. . . . . . . . . . . . 38

11 Courbes contrainte / déplacement hors-plan mesurées par les trois capteurs de déplacement (LVDT). . . . . . . . . . . . . . . . 39

12 a- Objet $A B$ correspondant à l'image $A^{*} B^{*}$ avec une mise au point optimale (i.e., sans défocalisation). b- Effet d'un déplacement hors-plan $w$ sur la taille de l'image $A^{\prime} B^{\prime *} \ldots \ldots . . \ldots 40$

13 Courbe contrainte / déformation transverse dans le domaine élastique obtenue à partir de mesures avec des jauges de déformation et par corrélation d'images. . . . . . . . . . . . . . . 41

14 Courbe contrainte / déformation longitudinale dans le domaine élastique obtenue à partir de mesures avec des jauges de déformation et par corrélation d'images avec correction de déplacement hors-plan. . . . . . . . . . . . . . . 42

15 Écart de linéarité pour différents points de mesure de déformation par jauge et par corrélation d'images dans le domaine élastique. Les lignes horizontales correspondent à l'écart quadratique moyen pour les deux moyens de mesure. . . . . . . . . . . . . . . 43 
16 Champs de déplacement mesurés par corrélation d'images (facteur d'amplification: $\times 38$ ) pour différentes valeurs de déformations longitudinales $(\ell=32$ pixels, $\delta=32$ pixels). . . . . . . 44 


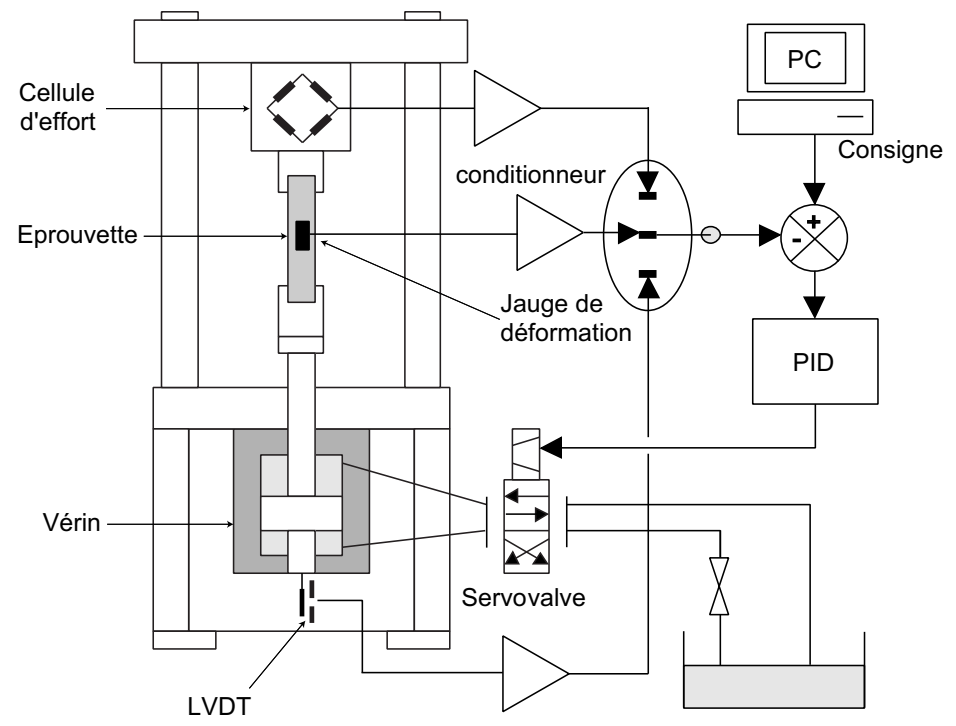

FIG. 1 - Schéma d'une machine electro-hydraulique asservie avec un pilotage en déformation. 


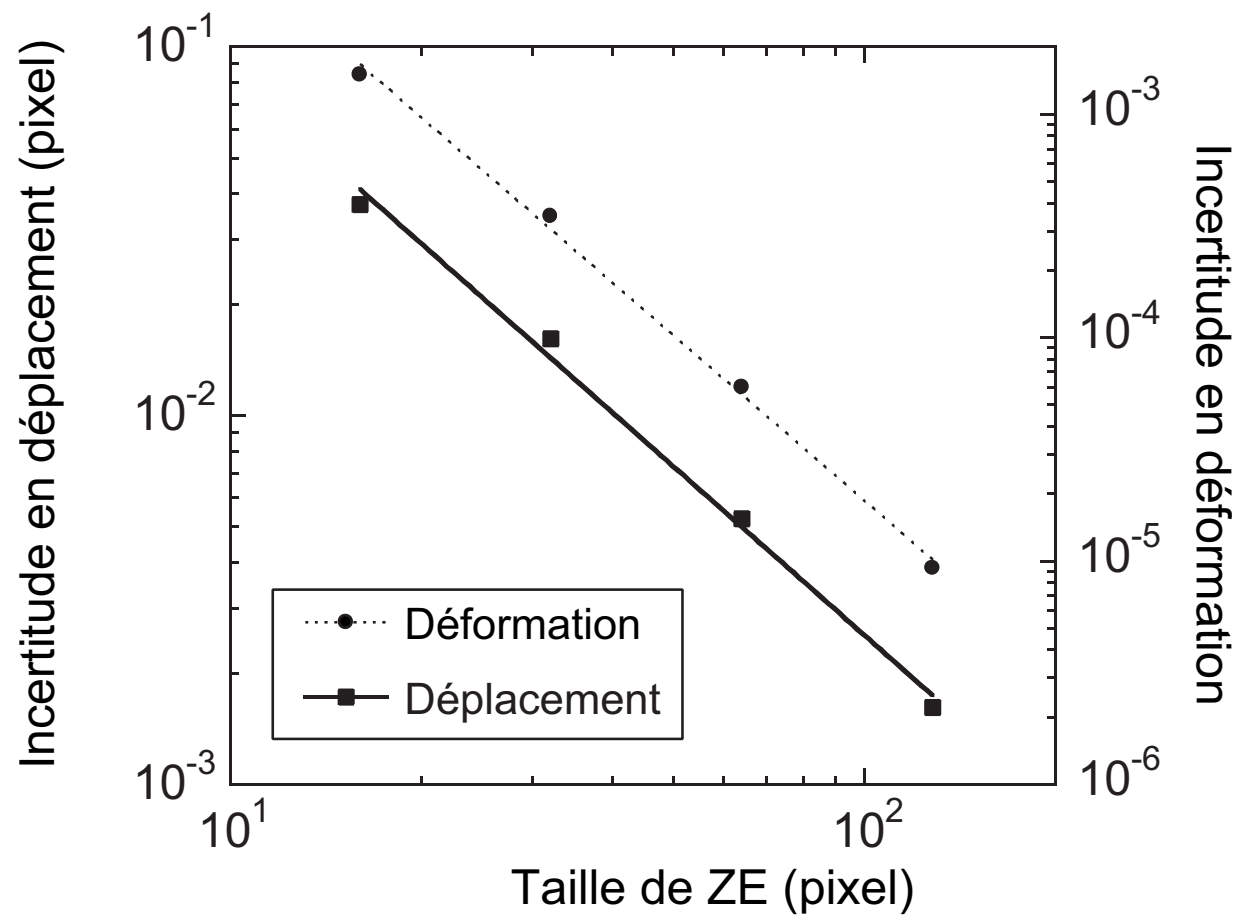

FIG. 2 - Incertitudes en déplacement et en déformation en fonction de la taille des zones d'étude. Les symboles pleins sont des résultats de corrélation. Les droites en trait continu correspondent à une interpolation en loi de puissance (-1.5) et en traits pointillés à une loi avec un exposant -2.5 . 

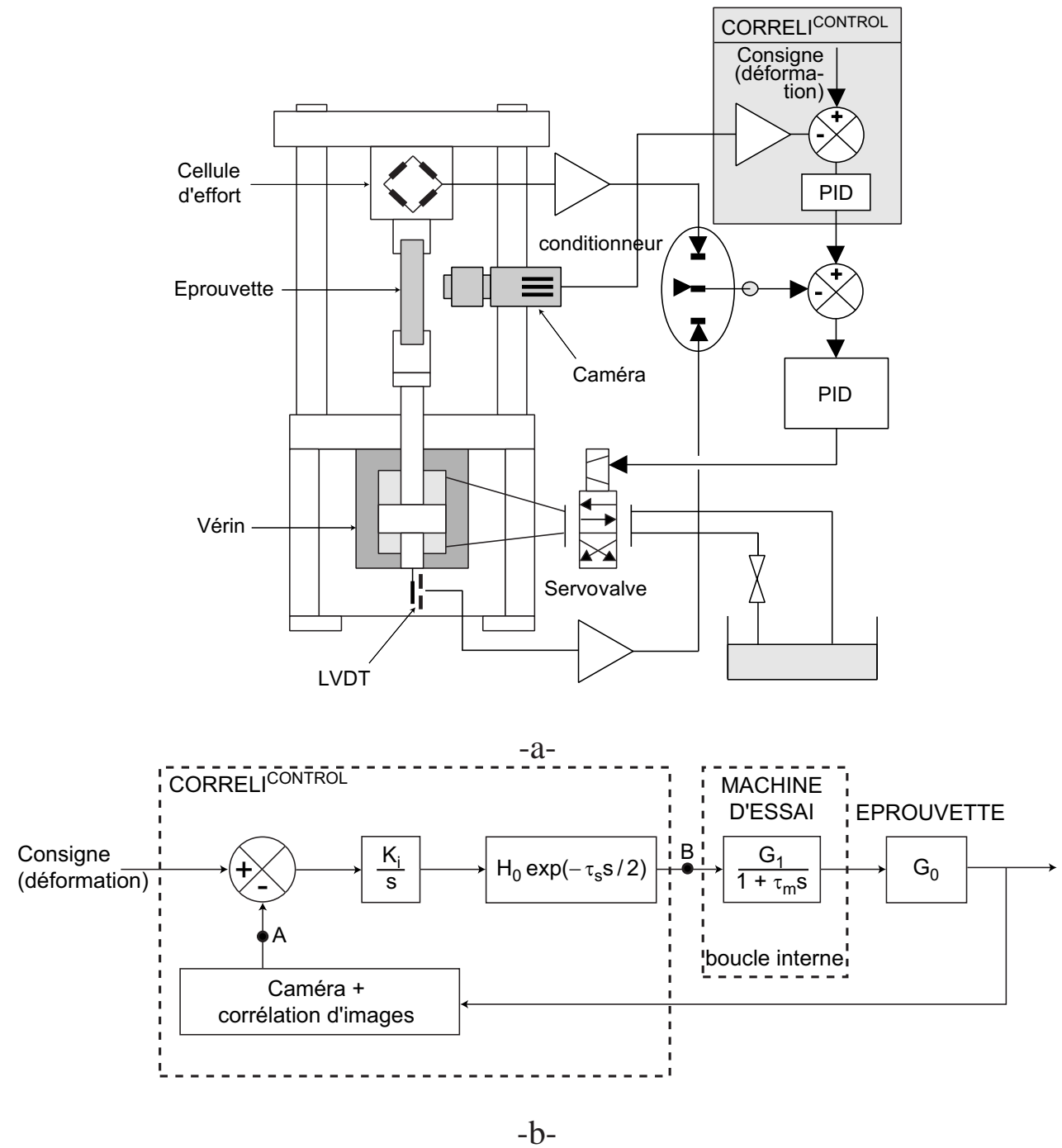

FIG. 3 - a-Schéma d'une implantation d'un asservissement en cascade utilisant la corrélation d'images pour le pilotage d'une machine électro-hydraulique asservie. b-Diagramme bloc d'un asservissement en cascade. Les points A et B correspondent aux signaux de la figure 6 

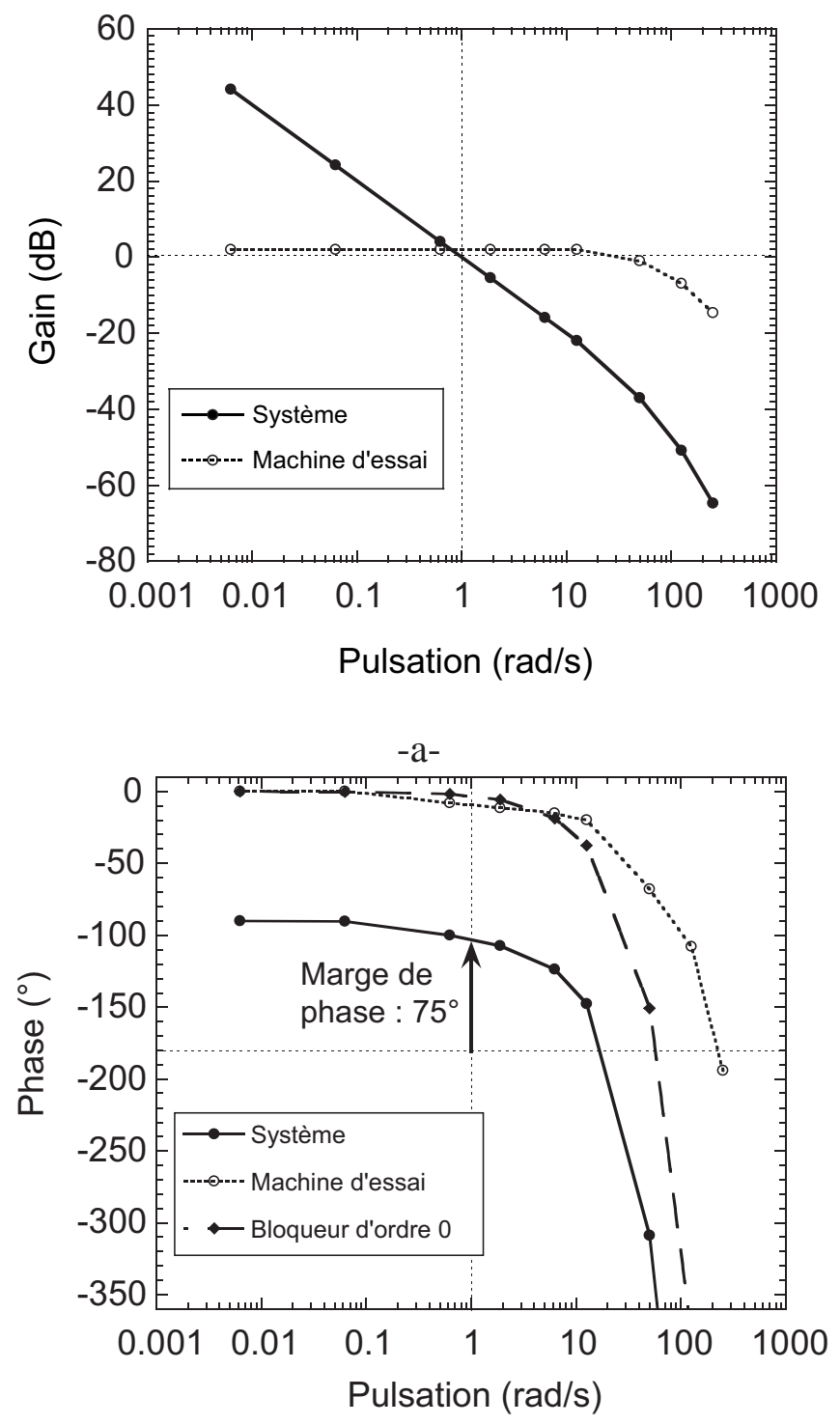

$-b-$

FIG. 4 - Diagrammes de Bode pour le système complet et la boucle intérieure (machine d'essai pilotée en déplacement). a-Gain en fonction de la pulsation. bPhase en fonction de la pulsation. Le déphasage induit par l'échantillonnage est également montré. 


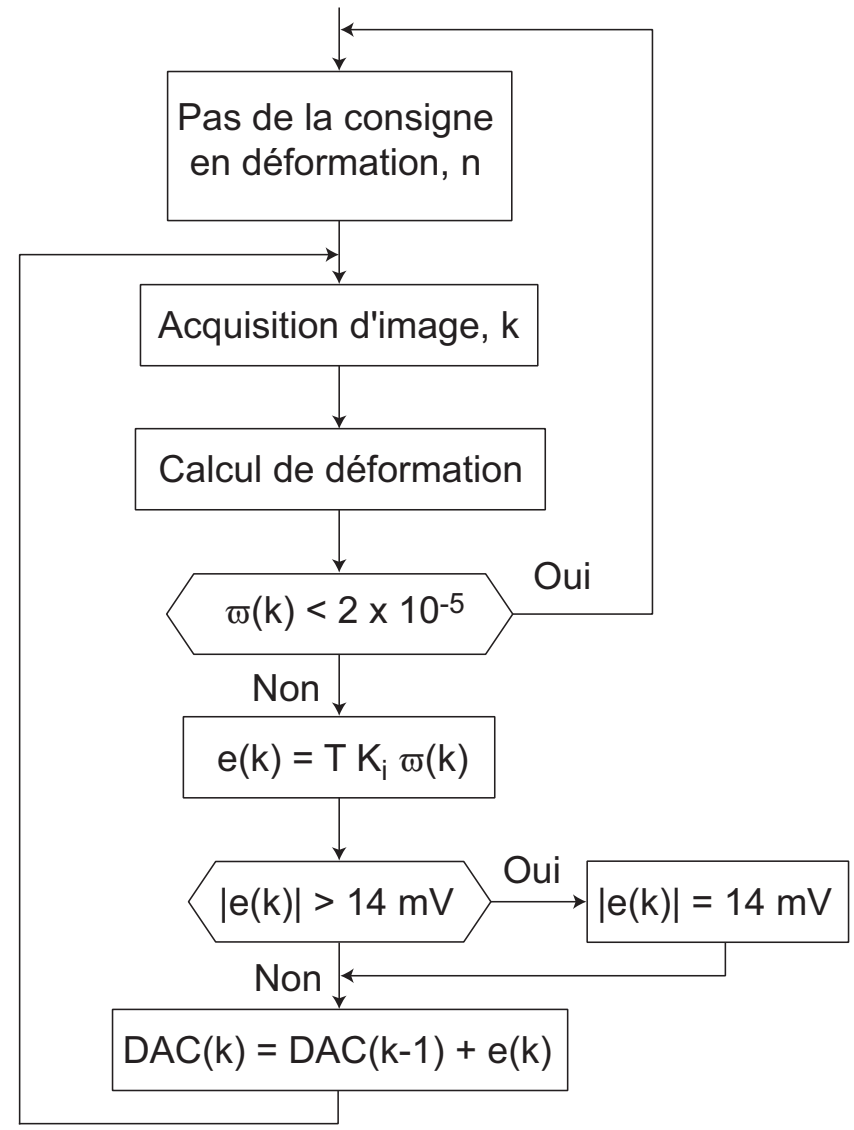

FIG. 5 - Organigramme de l'asservissement. 


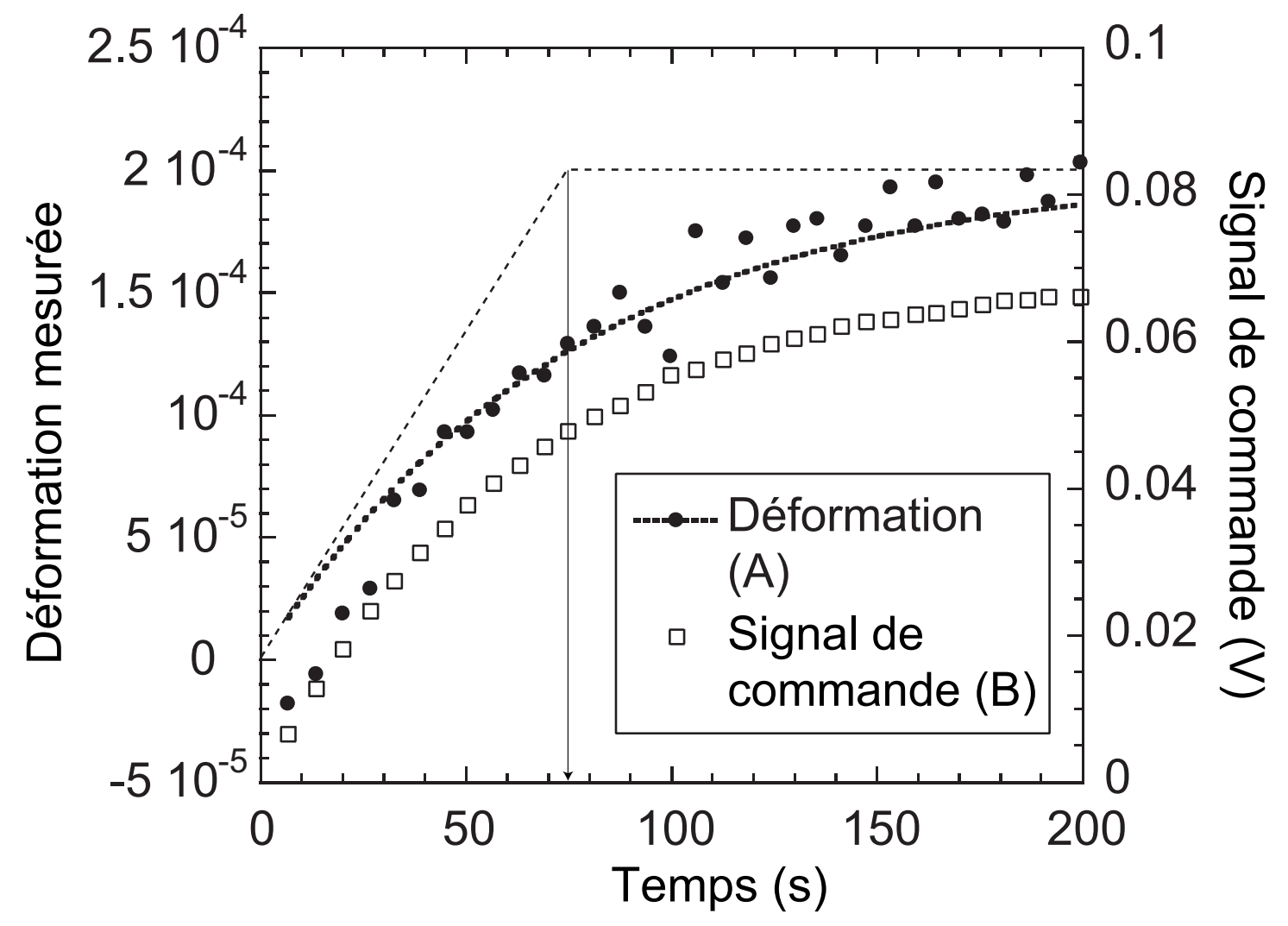

FIG. 6 - Réponse à un échelon du système et description par un premier ordre avec une constante de temps $\tau=75 \mathrm{~s}$. 


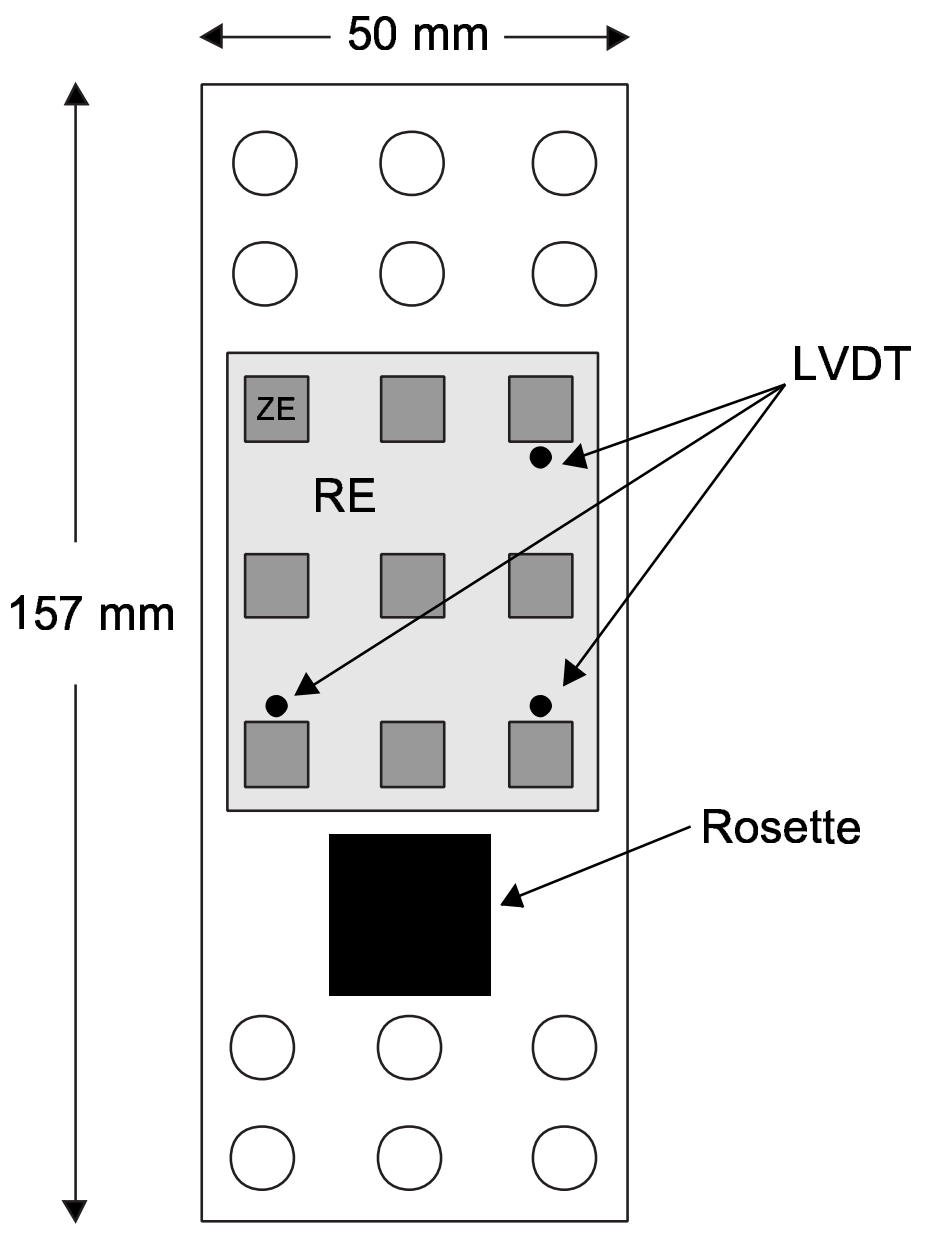

FIG. 7 - Géométrie de l'éprouvette et localisation des différents systèmes de mesure. 


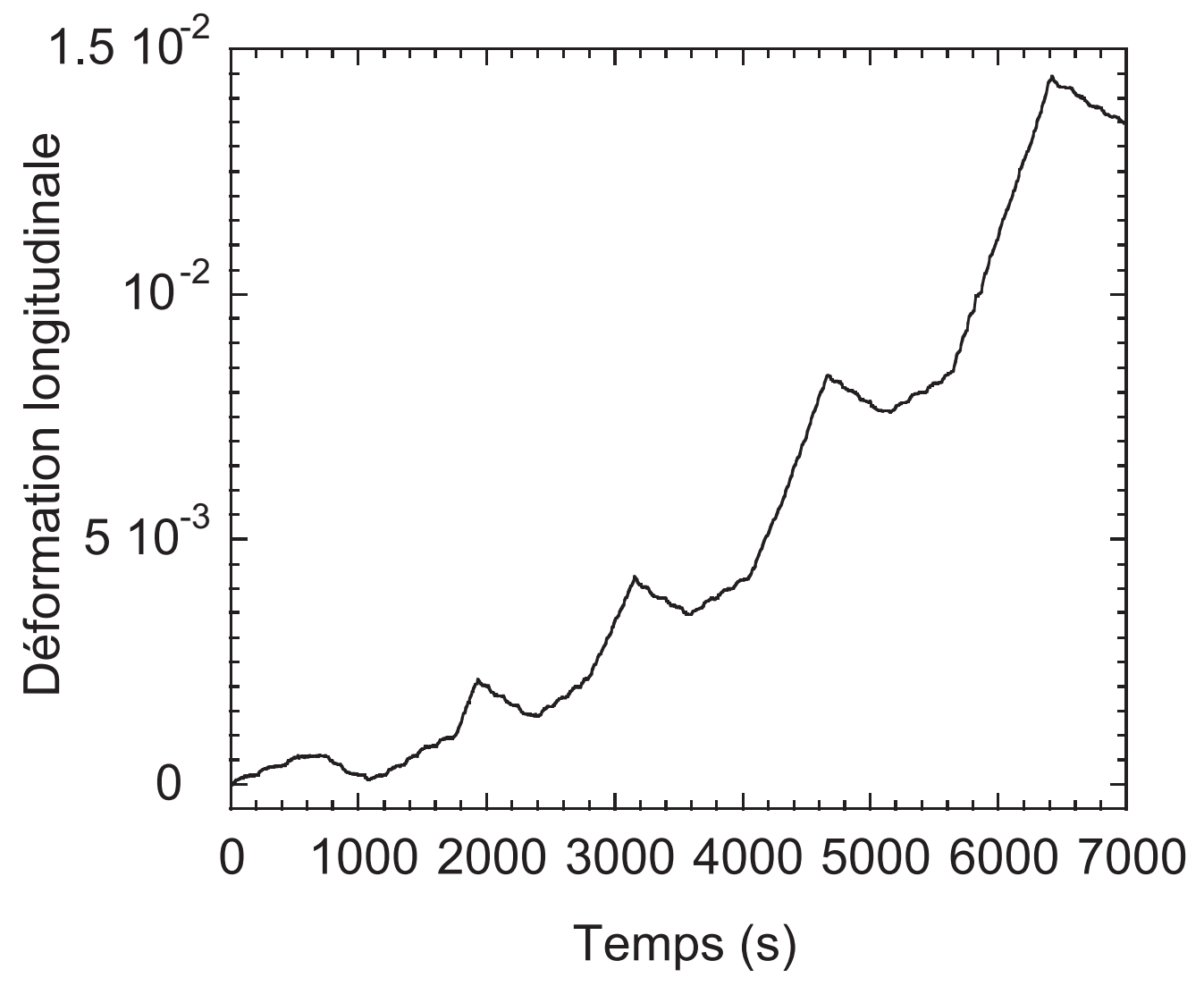

FIG. 8 - Déformation longitudinale en fonction du temps pour l'essai analysé. 


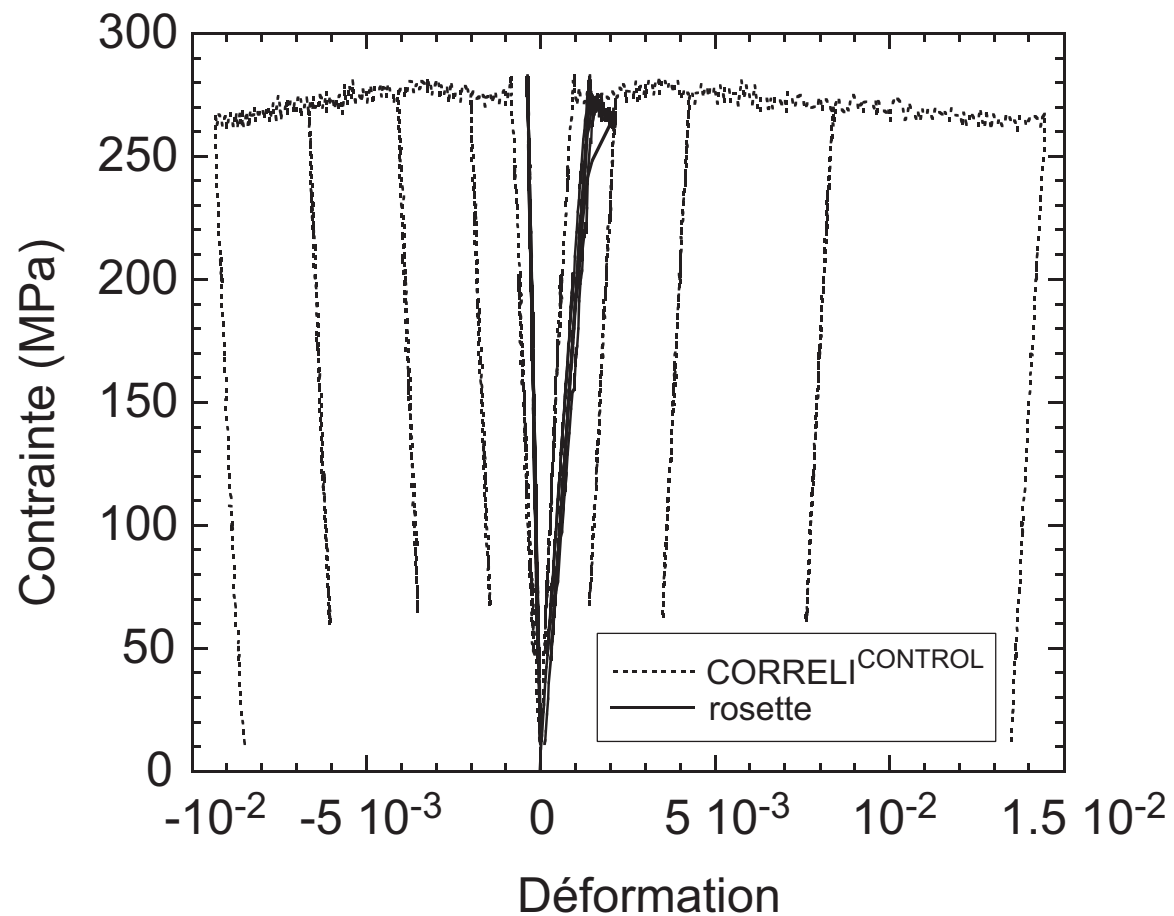

FIG. 9 - Courbe contrainte / déformations obtenue à partir de mesures avec des jauges de déformation et par corrélation d'images. 


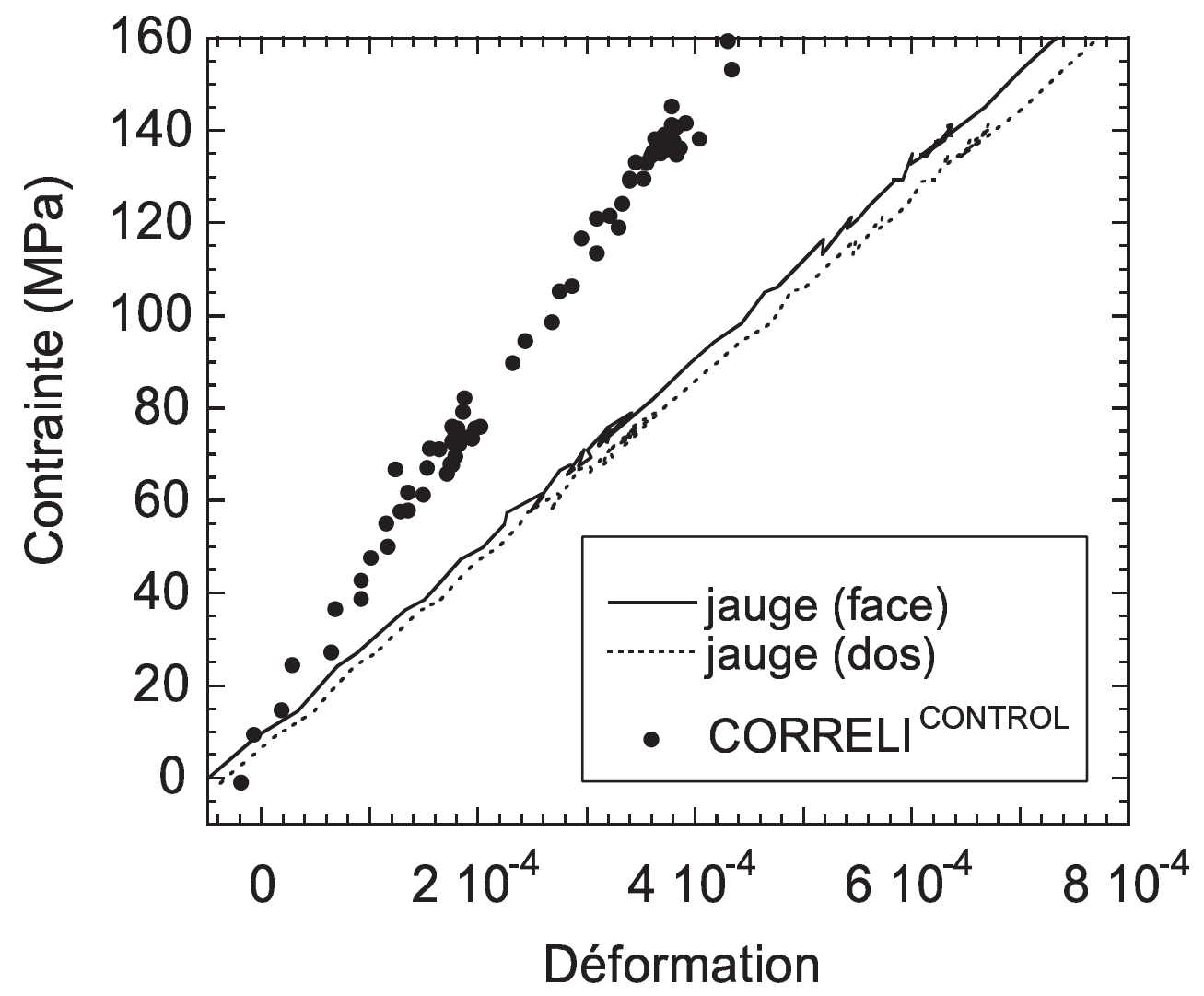

FIG. 10 - Courbe contrainte / déformation longitudinale dans le domaine élastique obtenue à partir de mesures avec des jauges de déformation et par corrélation d'images. 


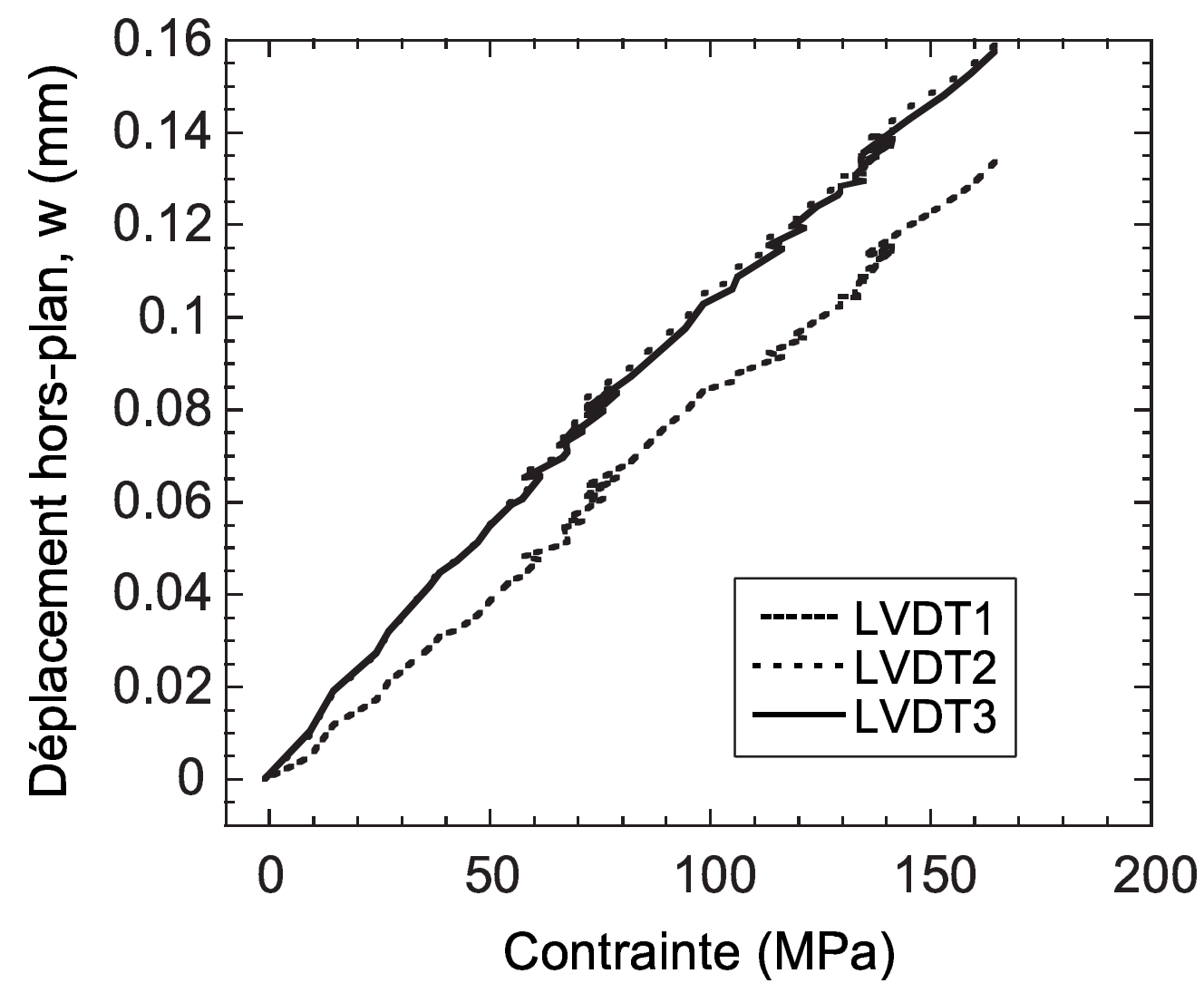

FIG. 11 - Courbes contrainte / déplacement hors-plan mesurées par les trois capteurs de déplacement (LVDT). 


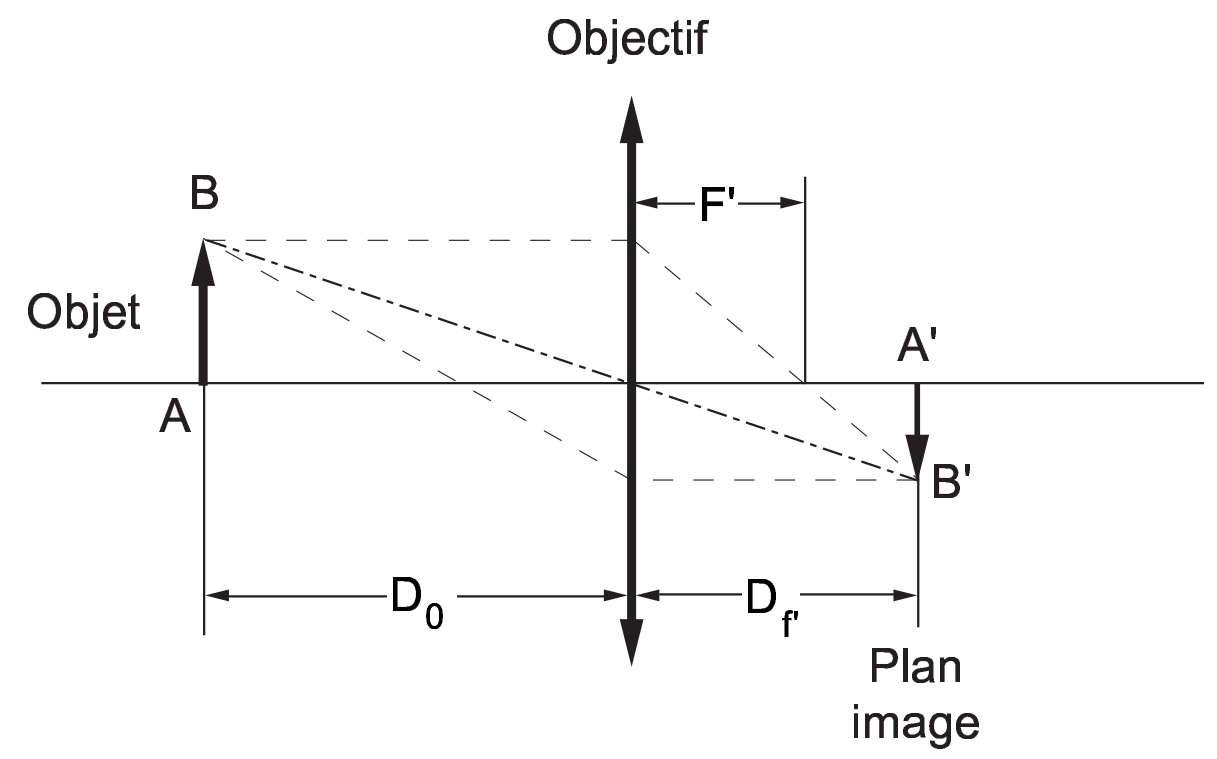

$-a-$

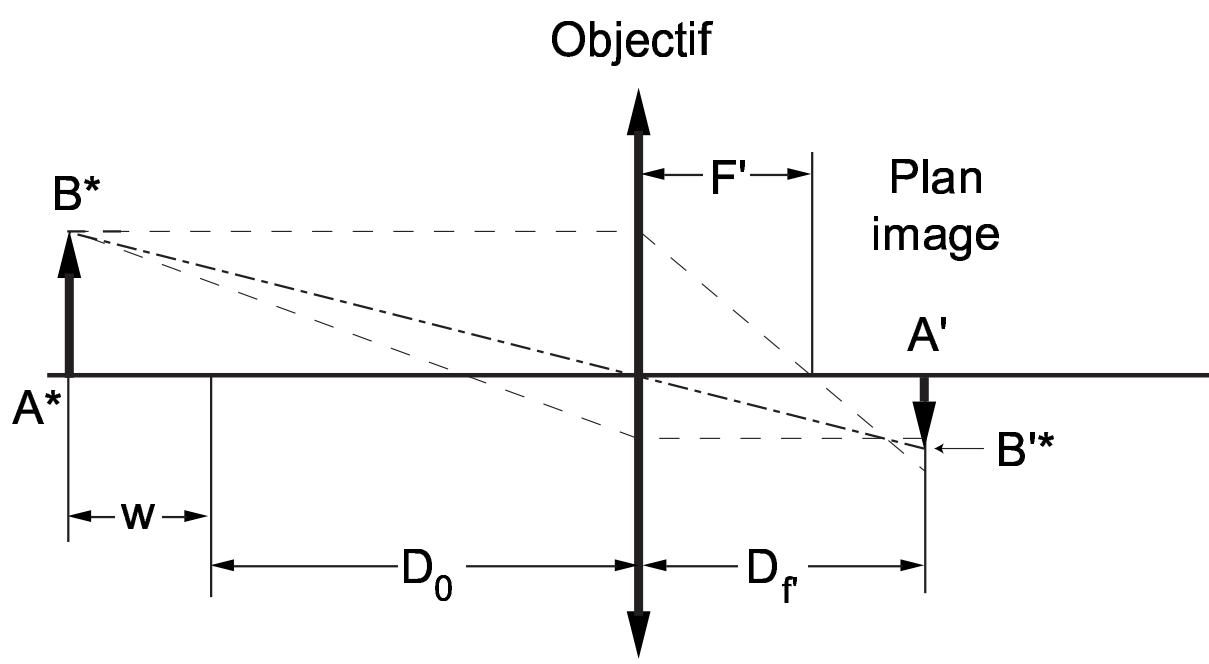

$-b-$

FIG. 12 - a-Objet $A B$ correspondant à l'image $A^{*} B^{*}$ avec une mise au point optimale (i.e., sans défocalisation). b- Effet d'un déplacement hors-plan w sur la taille de l'image $A^{\prime} B^{\prime *}$. 


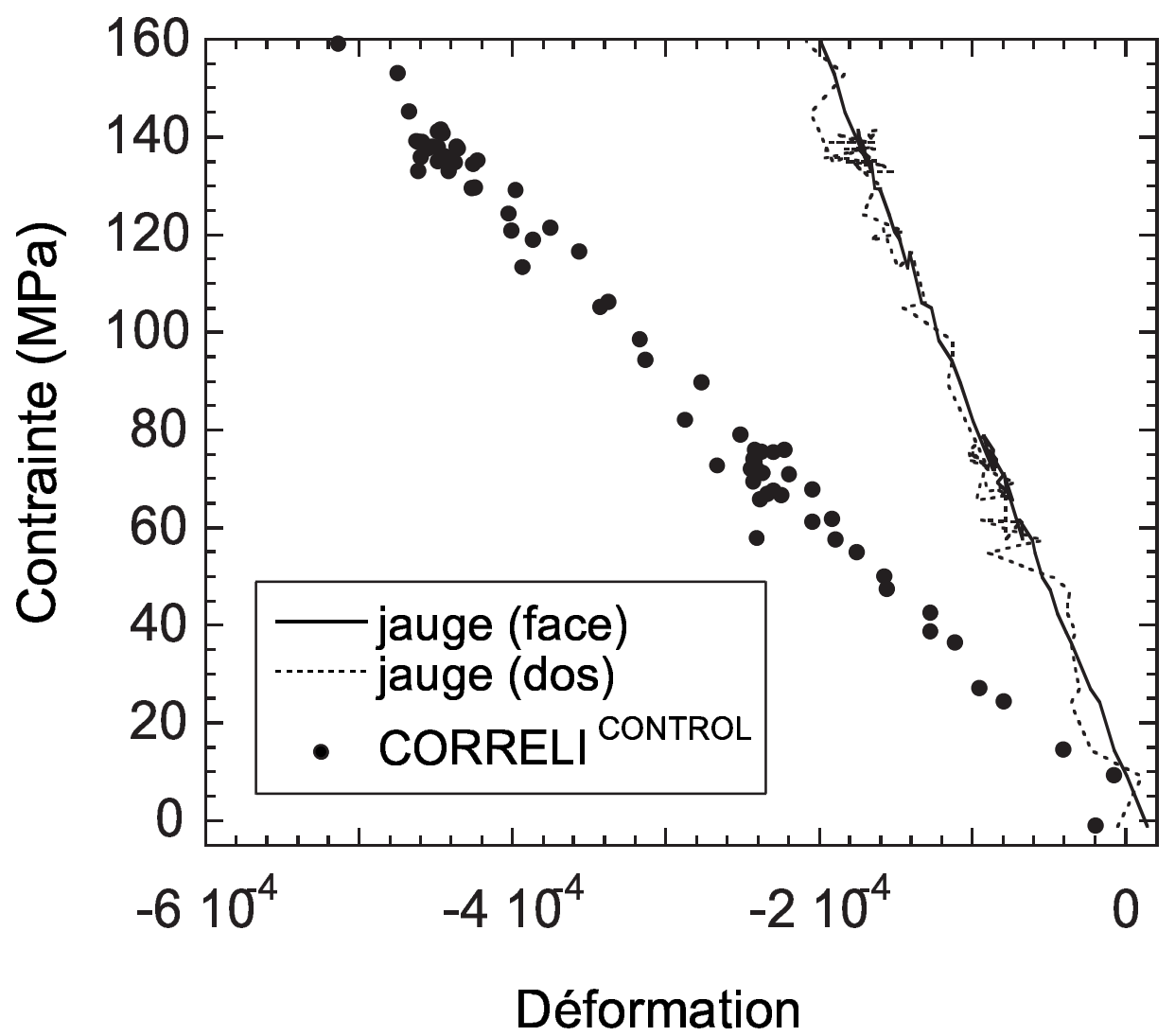

FIG. 13 - Courbe contrainte / déformation transverse dans le domaine élastique obtenue à partir de mesures avec des jauges de déformation et par corrélation d'images. 


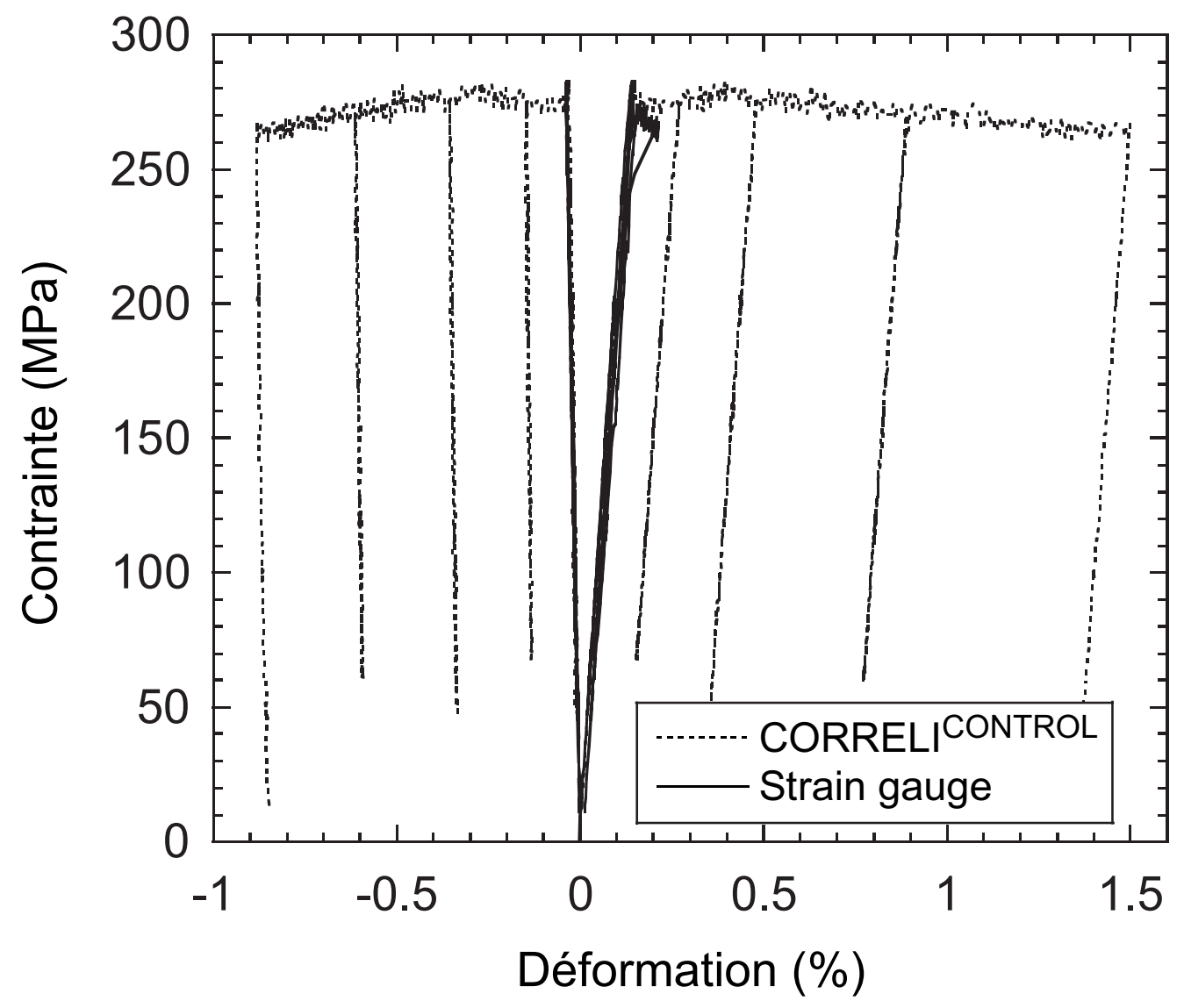

FIG. 14 - Courbe contrainte / déformation longitudinale dans le domaine élastique obtenue à partir de mesures avec des jauges de déformation et par corrélation d'images avec correction de déplacement hors-plan. 


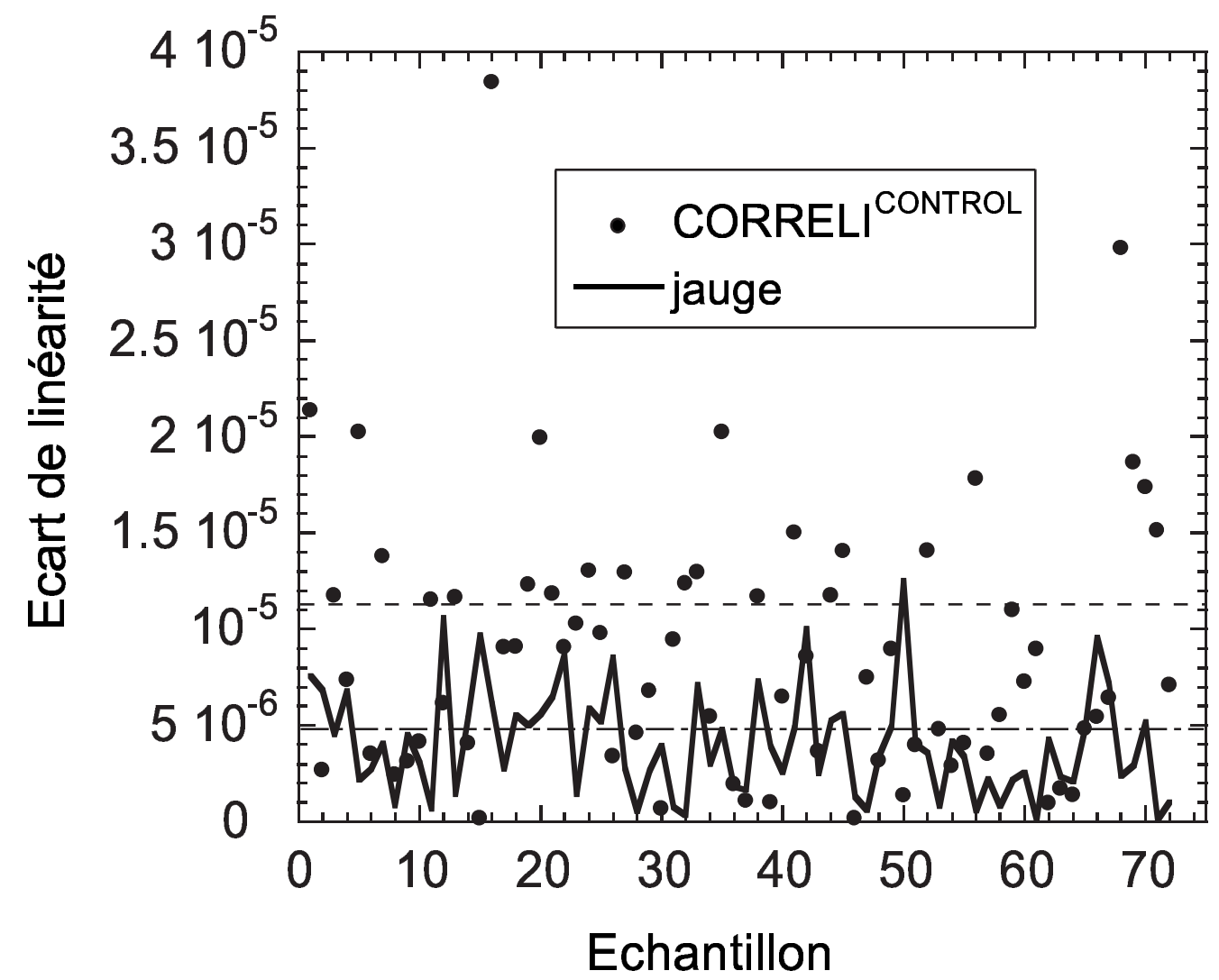

FIG. 15 - Écart de linéarité pour différents points de mesure de déformation par jauge et par corrélation d'images dans le domaine élastique. Les lignes horizontales correspondent à l'écart quadratique moyen pour les deux moyens de mesure. 

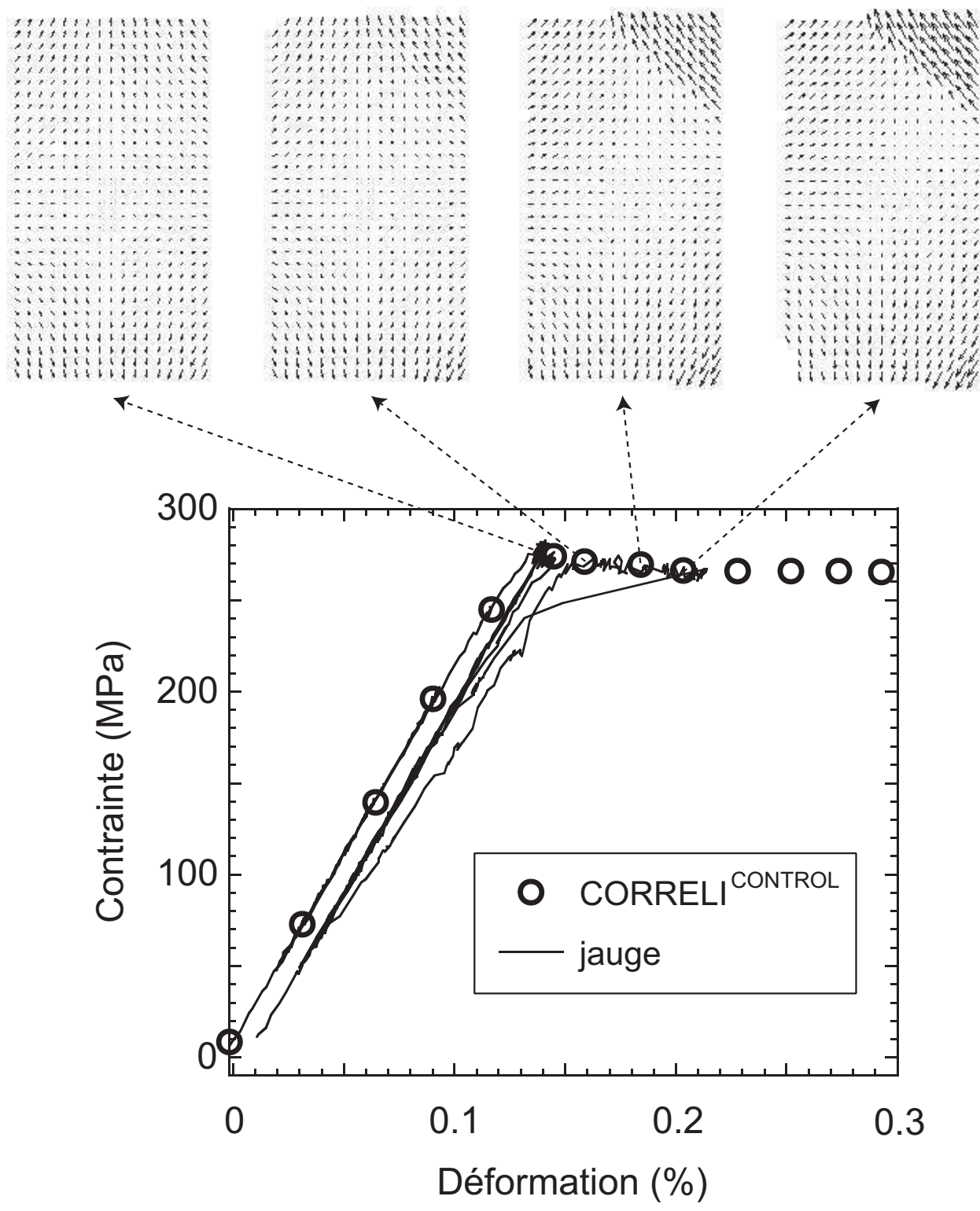

FIG. 16 - Champs de déplacement mesurés par corrélation d'images (facteur d'amplification: $\times 38)$ pour différentes valeurs de déformations longitudinales $(\ell=32$ pixels, $\delta=32$ pixels $)$. 